\title{
Side-Channel Protections for Picnic Signatures
}

\author{
Diego F. Aranha ${ }^{1}$, Sebastian Berndt ${ }^{2}$, Thomas Eisenbarth ${ }^{2}$, Okan Seker $^{2}$, \\ Akira Takahashi ${ }^{1}$, Luca Wilke ${ }^{2}$ and Greg Zaverucha ${ }^{3}$ \\ ${ }^{1}$ Department of Computer Science and DIGIT, Aarhus University, Denmark \\ \{dfaranha, takahashi\}@cs.au.dk \\ ${ }^{2}$ University of Lübeck, Germany \\ \{s.berndt, thomas. eisenbarth, okan. seker, l.wilke\}@uni-luebeck. de \\ ${ }^{3}$ Microsoft Research, USA \\ gregz@microsoft.com
}

\begin{abstract}
We study masking countermeasures for side-channel attacks against signature schemes constructed from the MPC-in-the-head paradigm, specifically when the MPC protocol uses preprocessing. This class of signature schemes includes Picnic, an alternate candidate in the third round of the NIST post-quantum standardization project. The only previously known approach to masking MPC-in-the-head signatures suffers from interoperability issues and increased signature sizes. Further, we present a new attack to demonstrate that known countermeasures are not sufficient when the MPC protocol uses a preprocessing phase, as in Picnic3.

We overcome these challenges by showing how to mask the underlying zero-knowledge proof system due to Katz-Kolesnikov-Wang (CCS 2018) for any masking order, and by formally proving that our approach meets the standard security notions of non-interference for masking countermeasures. As a case study, we apply our masking technique to Picnic. We then implement different masked versions of Picnic signing providing first order protection for the ARM Cortex M4 platform, and quantify the overhead of these different masking approaches. We carefully analyze the side-channel risk of hashing operations, and give optimizations that reduce the $\mathrm{CPU}$ cost of protecting hashing in Picnic by a factor of five. The performance penalties of the masking countermeasures ranged from 1.8 to 5.5, depending on the degree of masking applied to hash function invocations.
\end{abstract}

Keywords: side-channel attacks · masking $\cdot$ MPC-in-the-head $\cdot$ Picnic signatures

\section{Introduction}

As the possible advent of a quantum computer threatens the security of widely deployed cryptographic schemes, the design of new quantum-resilient alternatives is a pressing task. Motivated by this issue, the US National Institute for Standards and Technology (NIST) is currently holding the Post-Quantum Cryptography (PQC) Standardization Process, in which Round 3 "finalists" and "alternate candidates" have been recently announced. Among them is Picnic, a signature scheme $\left[\mathrm{ZCD}^{+} 20\right]$, which follows Ishai et al.'s MPC-in-the-head (or MPCitH, short for multi-party computation in-the-head) paradigm for constructing zero-knowledge (ZK) proof systems [IKOS07]. One of the attractive features of MPCitH-style signatures is that they can be constructed without number-theoretic hardness assumptions, and rely only on the security of symmetric-key primitives. Concretely, following the standard Fiat-Shamir paradigm [FS87], signatures in the MPCitH paradigm can be proven secure in the random oracle model, as long as the underlying hash function and block cipher are secure. Quoting [Nat20], "NIST also sees 
Picnic's reliance on only assumptions about symmetric primitives as an advantage in case the need arises for an extremely conservative signature standard in the future".

MPC-in-the-head vs probing side-channel analysis. Yet, despite such advantages with regard to the underlying assumptions, implementations of MPCitH-type signatures are not immune to side-channel attacks that threaten unprotected software. Recently, Gellersen et al. [GSE20] and Seker et al. [SBWE20] (which, to the best our knowledge, are the only previous works that considered side-channel security of MPCitH) observed that a direct implementation of MPCitH signing is susceptible to a probing side-channel attack, which is in practice realized by, e.g., differential power analysis (DPA). Their proposed attack is devastating - it allows a side-channel attacker to successfully recover the secret signing key, after observing 30 signatures. To understand their attack, it is useful to review the underlying "commit-and-open" approach typically used by MPCitH proofs. The attacks target the three-round ZK proof system underlying some Picnic instances, known as ZKB $++\left[\mathrm{CDG}^{+} 17\right]$ (an optimized variant of ZKBoo [GMO16]).

MPCitH proof systems like $\mathrm{ZKB}++$ follow a typical commit, challenge, response structure (which, in fact, can be seen as a $\Sigma$-protocol [Dam10]), and prove knowledge of a witness $\mathbf{w}$ that satisfies a statement for a hard relation. For example, we can prove knowledge of a key (the witness) that relates a plaintext-ciphertext pair (the statement) for a block cipher (the relation). The ZKB++ proof system is built on a three-party MPC protocol $\Pi_{f}$ for a function $f$ implementing this hard relation (the block cipher in our example). To initialize the protocol, the prover $\mathrm{P}$ first additively secret-shares the witness such that $\mathbf{w}=\mathbf{w}_{1}+\mathbf{w}_{2}+\mathbf{w}_{3}$, and considers each share $\mathbf{w}_{i}$ as a private input to party $P_{i}$. Then $\mathrm{P}$ runs $\Pi_{f}$ "in the head", i.e., it simulates a run of the protocol between the three parties, and produces for each $P_{i}$ a commitment to its view, i.e., the input $\mathbf{w}_{i}$, output, communication, and random tape. These three commitments are sent to the verifier $\mathrm{V}$, who replies with a challenge, for $\mathrm{P}$ to open two of them. Finally, $\mathrm{V}$ checks the openings for consistency and for an accepting output. The (honest verifier) ZK property of the protocol is guaranteed by the 2-privacy of $\Pi_{f}$, i.e., it is possible to simulate up to 2 parties' views given their inputs, and the output of the protocol execution.

But once a side-channel adversary probes the unopened party's view, in particular their share of the witness, the adversary can recover the secret witness. To thwart this devastating probing attack, Seker et al. [SBWE20] proposed the SNI-in-the-head (SNIitH) approach, which naturally retains the privacy of the MPC protocol, but also adds strong non-interference $(\mathrm{SNI})\left[\mathrm{BBD}^{+} 16\right]$ security, which is a strong provable security notion for ISW-style masking countermeasures (as defined by Ishai-Sahai-Wagner [ISW03]). In the SNIitH approach, the number of parties is generalized to $N$ and the underlying MPC protocol is assumed to have $(N-1)$-privacy, so that the views of up to $N-1$ parties may be safely revealed. However, instead of opening $N-1$ parties as a typical MPCitH prover would, an SNIitH prover only reveals $N-t-1$ parties as a response, where the parameter $t$ serves as the "buffer" for probing security. This way, the prover makes sure that at least one party's internal state remains completely hidden, even if the side-channel adversary observes up to $t$ variables during the execution of MPC protocol $\Pi_{f}$. Relying on this idea, Seker et al. generalized $\mathrm{ZKB}++$, and gave a variant of Picnic that is provably secure against probing adversaries. However, as we shall see below, there are still several practical challenges which seem hard to overcome with SNIitH. In this paper, we push forward the study of side-channel resistant MPCitH signing, to tackle these open questions.

Maintaining the verification algorithm and signature size. When applied to Picnic, the SNIitH approach changes the number parties and the number of opened views, and the result is essentially a different parameter set. Accordingly, the verification algorithm has to be modified, and now depends on the masking order. For example, the signer would have to simulate a five-party MPC to achieve first order protection (i.e., $N=5, t=1$ ). Hence, the verifier needs to check the consistency of 3 -out-of- 5 views, instead of 2-out-of-3 
as in the original Picnic/ZKB++ scheme. This is a major drawback in practice, since the introduction of side-channel protection would immediately break interoperability with existing verification algorithms. In order to support signers with varying levels of sidechannel protections, verifiers would have to be prepared to accept multiple combinations of parameters $(N, t)$. A more flexible design would allow different signers to set their level of side-channel protection independent of the verification software and costs. Moreover, the SNIitH approach inevitably sacrifices the soundness of underlying ZK proof system, since a malicious prover has a higher cheating probability when the number of views the verifier checks is smaller than $N-1$. Therefore to maintain the same security level as in the original proof system, the SNI-in-the-head approach must simulate more MPC instances, leading to larger signature sizes, and slower signing and verification times. In this work we design countermeasures without these drawbacks (as has been done for some other PQ signature schemes $\left.\left[\mathrm{BBE}^{+} 18, \mathrm{MGTF} 19, \mathrm{BBE}^{+} 19, \mathrm{GR} 19\right]\right)$.

Masking MPCitH with preprocessing. To the best of our knowledge, no previous work explored the possibility of masking the newer MPC-in-the-head with preprocessing (MPCitH-PP) paradigm of Katz, Kolesnikov, and Wang (KKW, [KKW18]), which produces much more compact proofs (and shorter signatures in turn). KKW is used in the latest version of Picnic, Picnic3 [KZ20], and in a similar signature scheme BBQ [dDOS19]. The preprocessing phase is independent of the witness, and is used by the parties to establish correlated random values, such as multiplication triples, that they can use during the MPC protocol to improve efficiency. Since it happens before the main, witness-dependent MPC protocol, the preprocessing phase is also called an offline phase, and the main part is called the online phase. We first observe that the preprocessing phase provides additional attack surface not present in MPCitH protocols without preprocessing. In the KKW protocol, the prover first simulates many instances of the MPC protocol consisting of offline and online phases, and commits to each party's view in both phases. Then for each instance of the MPC protocol, the prover opens either the offline phase or the online phase, depending on the challenge sent from the verifier. To open the offline phase, the prover reveals the views of all $N$ parties (which is secure, since preprocessing views are independent of the witness). For the online phase, the prover reveals the views of $N-1$ parties (which is secure by the $N-1$ privacy of the MPC protocol). In the former scenario, since the offline phase contains the correlated random values used by all parties during the online phase, a probing adversary can break the privacy of the underlying MPC protocol by probing values from the corresponding online phase (which is computed by the prover but not revealed).

We note that the attack succeeds for any number of opened views, since MPCitH-PP inherently relies on revealing all views for the offline phase. This indicates that the SNIitH approach cannot mitigate this type of probing attack. Given all this, we are motivated to design an alternative countermeasure addressing the following question:

Can we mask signature generation in signature schemes constructed with the $M P C$-in-the-head-with-preprocessing paradigm in a provably secure manner, without modifying the verification algorithm?

\subsection{Contributions}

Side-channel attacks against Picnic3 and KKW. We confirm experimentally that our new probing attack described above indeed applies to Picnic3, by measuring the leakage of the (unmasked) reference code on the Cortex M4. In Section 3 we elaborate on the high-level attack strategy for MPCitH-PP and describe how to mount a practical signing key recovery attack against Picnic3, as well as any three-round KKW-type protocol. Fig. 1 experimentally shows this attack is practical.

Masking MPC-in-the-head with preprocessing. In Section 4 we show how to mask the KKW proof system for any masking order. Our first observation is that all building 
blocks in both the offline and online computations can be masked with existing SNI-secure gadgets without changing the number of opened parties. The main technical difficulty is that the prover opens a different subset of sensitive information, depending on the challenge. Nevertheless, we are able to present a solution achieving strong non-interference of the basic building blocks of the KKW proof system. Then together with a known generic composition result $\left[\mathrm{BBD}^{+} 16\right]$, we prove that our masked $\mathrm{KKW}$ prover algorithm for the commit and response phases satisfies non-interference with public output (NIo) security $\left[\mathrm{BBE}^{+} 18\right]$ for all possible public outputs. The CPU cost of masking follows the common pattern where nonlinear operations have cost $O\left(T^{2}\right)$, and all other operations are linear in $T$, where $T$ denotes the masking order. In addition to giving formal proofs of the (strong) non-interference of our solution, we also use the maskVerif tool $\left[\mathrm{BBD}^{+} 15 \mathrm{a}, \mathrm{BBD}^{+} 16\right]$ to verify our conclusions. We stress that our variant outputs a proof identical to that of the unprotected $\mathrm{KKW}$, so it is interoperable with existing verification operations.

Masked implementation of Picnic3. Following our generic masking technique from Section 4, we present an NIo-secure masked implementation of Picnic3 for NIST security level L1 with first-order protection, and we report concrete experimental results on the low-end ARM Cortex-M4 STM32F4 platform (see Sections 5 and 6). The complete specification of NIo-secure Picnic3 is presented in Appendix C.1. The figures were collected from a port of the optimized version of the Picnic3 reference code, with the help of the pqm4 [KRSS] framework. However, the overhead incurred by provably secure masking is expensive - signing time is increased by 5.5x. Using this provably secure implementation as a baseline, we performed a number of optimizations described in Section 5, especially to improve hashing performance. Since a large part of the signing time in Picnic3 is spent hashing (e.g., 70\% on the M4), we carefully analyzed which hashing operations must be masked, and found that the majority may remain unmasked. We then show that since all calls have either a non-sensitive input or output, a weaker form of masking is sufficient (under a mild assumption), further reducing the cost. Taken together, these optimizations reduce the cost of protected hashing significantly, and we observed overhead ranging from $1.8 \mathrm{x}$ to $2.8 \mathrm{x}$ (depending on the type of protections applied to hash function invocations). Since our hash function optimizations do void the provable side-channel-security of the signature scheme implementation as a whole, we experimentally verified the absence of leakage, to support our arguments that security is maintained in practice.

Masked implementation of SHA-3. We also implemented a masked version of SHA-3, optimized with M4 assembly, as none was freely available. As SHA-3 is common to many PQ schemes and the M4 is a common embedded target, we expect this will be of independent interest. The implementation supports a range of options, from slower but provably SNI-secure, to our much faster optimized options. We experimentally verified that there was an absence of leakage in the optimized version.

Our first-order masked Picnic3 and SHA-3 implementations, as well as formal verification scripts, are publicly available under crypto_sign/picnic311/masked/ of the Picnic-M4 GitHub repository. ${ }^{1}$

\subsection{Related work}

Since the seminal work by Ishai et al. [IKOS07] the MPCitH paradigm has been actively studied over the past decade. In particular, two closely related protocols ZKBoo [GMO16] and $\mathrm{ZKB}++\left[\mathrm{CDG}^{+}{ }^{17}\right]$ brought $\mathrm{MPCitH}$ closer to practice, leading to the submission of Picnic1 to Round 1 of the NIST PQC Standardization Process. Katz, Kolesnikov, and Wang [KKW18] extended the paradigm to MPCitH-PP and corresponding version, Picnic2, was added during Round 2. Kales and Zaverucha [KZ20] further optimized Picnic2 from various implementation aspects and accordingly proposed Picnic3. Although our

\footnotetext{
${ }^{1}$ Found at https://github.com/dkales/picnic_m4.
} 
masked implementation focuses on Picnic3, which is instantiated with KKW and the LowMC circuit $\left[\mathrm{ARS}^{+}\right.$15], our generic approach in Section 4 also applies to BBQ (KKW instantiated with the AES circuit) [dDOS19] and Baum and Nof's variant of KKW (instantiated over an arithmetic circuit for proving SIS instances) [BN20]. A similar offline-online paradigm also appears as a notion called "sigma protocol with helper" [Beu20], used to construct proof of knowledge for systems of quadratic equations, improving a protocol of Stern [Ste94].

The stateful hash-based signature schemes XMSS and LMS are known to be relatively resistant to side-channel attacks, as they basically use pseudorandom keys for each signature. Hence, their resistance against side-channel attacks rests on the resistance of the underlying pseudorandom number generator [EvMY14, $\left.\mathrm{KGB}^{+} 18\right]$.

Comparison with other NIST PQC candidates. To the best of our knowledge, most other PQ signatures currently do not have publicly available masked implementations, except for lattice-based Fiat-Shamir with aborts [Lyu09] signatures: GLP [BBE ${ }^{+}$18], BLISS [BBE ${ }^{+}$19], Dilithium [MGTF19] (NIST PQC finalist) and qTESLA [GR19] (Round 2 candidate). While these masked signing operations do output a signature compliant with the existing verification algorithm, they rely on an additional non-standard hardness assumption for provable side-channel-security (see $\left[\mathrm{BBE}^{+} 18, \mathrm{DOTT} 21\right]$ for details). The issue could be circumvented by modifying the "commit" message of the underlying $\Sigma$ protocol, but this in turn breaks the interoperability of the output signature. By contrast, signatures directly derived from our generic approach to masking KKW (Section 4) as well as NIo-secure Picnic3 implementation maintain interoperability, and may optionally make additional assumptions for improved performance. Performance-wise, the benchmarks on Cortex M4 given by Gérard and Rossi [GR19, Table 6] show much less overhead than ours: their first-order protected qTESLA-I incurs only $2.1 \mathrm{x}$ overhead in signing clock cycles and requires $343 \mathrm{~KB}$ of fresh randomness, while provably secure masked Picnic3 is $5.5 \mathrm{x}$ slower than unprotected and consumed $158 \mathrm{MB}$ of randomness. However, by trading provable security guarantee as we describe in Section 5, our empirically validated countermeasures achieve a lower overhead overall of $1.8 \mathrm{x}$, requiring $2 \mathrm{MB}$ of randomness. Giving a meaningful performance comparison with masked Dilithium [MGTF19] is hard, as they only provide benchmarks on Intel for the whole signing operation. Although their overhead for first-order protection is about 5.6x, we expect that it can be made faster on the M4 by using the platform's TRNG.

\section{Preliminaries}

Notation. We denote the set $\{1, \ldots, T\}$ by $[T]$. The number of multiplication gates in a circuit $C$ is denoted by $|C|$.

Security levels. The parameter sets for the algorithms submitted to the NIST competition must meet one of five security levels. Picnic defines parameters for security levels L1, L3 and L5, corresponding to the security of AES 128, 192 and 256, respectively. For instance, parameters at level L1 aim to provide 128-bit security against classical attacks.

LowMC. The LowMC block cipher is described in detail in Appendix F, and here we briefly review the notation. The block and key size are both $n$ bits, the number of rounds is denoted $r$, and the number of S-boxes per round is denoted $s$. In the Picnic3 parameters, $n=3 s$, since the three-bit S-box is applied to the full state. There are also constants: $K_{i}$ are matrices used to compute the round keys; $L_{i}$ are matrices used for the linear layer, and $R_{i}$ are vectors used as round constants.

Formal Verification. In order to check our formal security analysis, we use the tool maskVerif developed by Barthe et al. $\left[\mathrm{BBC}^{+} 19\right]$. The tool provides an automatic and formal security verification of higher-order masking implementations based on the NI and SNI notions. Briefly speaking, it checks every possible attack combination within 
the implementation and either provides a security proof for the specified order or gives a list of potential attack targets in the implementation. The bottleneck of the tool is the order of the masking and the complexity of the implementation. It has been used in the literature to provide assurance of masked implementations $\left[\mathrm{BBD}^{+} 15 \mathrm{a}, \mathrm{GSDM}^{+} 19, \mathrm{SEL} 21\right]$. In this work, we use maskVerif to check SNI security of the basic components (namely, multi-party computation of multiplication in both online and offline phases) of the KKW proof system up to order 4 .

Leakage analysis. In this work we follow the test vector leakage assessment (TVLA) method by Goodwill et al. [GJJR11], which is based on Welch's $t$-test. TVLA implements a pass-fail test to decide if an implementation has exploitable leakage. It detects leakage at a given order and has two different versions: the non-specific and the specific method. The first version is defined as fixed-vs-random (FvR) and it aims to detect all possible sources of first-order leakage. During the trace collection phase, a set of side-channel traces is collected by processing either a fixed input or a random input under the same conditions. If the $t$-test only gives a very small value, this indicates that the run of the algorithm on a fixed input is indistinguishable from a run of the algorithm on a random input. Hence, a small value in the fixed-vs-random scenario implies the absence of sensitive leakage. The second test is defined as random-vs-random (RvR), and employs only traces with a random input using a function of inputs to sort the traces. The main advantage of the RvR method is that it can identify specific sources of exploitable leakage and thus shows the feasibility of an actual attack.

After collecting and sorting the traces, the means $\left(\mu_{0}, \mu_{1}\right)$ and standard deviations $\left(\sigma_{0}, \sigma_{1}\right)$ for the two sets are calculated. Welch's $t$-test is computed as $t=$ $\left[\mu_{f}-\mu_{r}\right] /\left[\sqrt{\left(\sigma_{f}^{2} / n_{f}\right)+\left(\sigma_{r}^{2} / n_{r}\right)}\right]$, where $n_{f}$ and $n_{r}$ denote the number of traces for the sets, respectively. The $t$-test indicates whether the two distributions are indistinguishable for a first-order side-channel analysis. We apply the customary threshold values for long traces suggested by $\left[\mathrm{DZD}^{+} 18\right]$. We use the value 5.7 for traces of length more than $10^{4}$, and the value 6.1 for traces of length more than $10^{6}$. The threshold rejects the null-hypothesis of non-leakage with $>99.99 \%$ probability.

\subsection{MPC-in-the-head with preprocessing}

MPC-in-the-head. We first describe the basic approach to construct a zero-knowledge proof of knowledge (ZKPoK) system for an arbitrary nondeterministic polynomial-time (NP) language $L$, following Ishai et al. [IKOS07] and its generalization due to Giacomelli et al. [GMO16]. Given $L$, we can define an $N P$ relation $R(\mathbf{x}, \mathbf{w})$ which returns 1 if its input consists of a valid pair of statement $\mathbf{x} \in L$ and corresponding witness $\mathbf{w}$, and outputs 0 otherwise. An MPCitH proof system $(\mathrm{P}, \mathrm{V})$ is built upon some $N$-party MPC protocol that jointly computes a function $f$, where $f$ takes $\mathbf{x}$ and $\mathbf{w}$ as public and private input, respectively, and outputs $f_{\mathbf{x}}(\mathbf{w})=R(\mathbf{x}, \mathbf{w})$. For example, for a given encryption algorithm Enc of a block cipher like LowMC $\left[\mathrm{ARS}^{+} 15\right]$, one can define $f_{\mathbf{x}}(\mathbf{w}):=\operatorname{Enc}(s k, p) \stackrel{?}{=} c$, where the statement $\mathbf{x}=(p, c)$ is a plaintext-ciphertext pair, and witness $\mathbf{w}=s k$ is a private encryption key, respectively. In this case, the prover $\mathrm{P}$ proves knowledge of a private key that produces a certain public ciphertext from the corresponding public plaintext.

At a high level, an MPCitH prover $\mathrm{P}$ attempts to convince the verifier $\mathrm{V}$ that they hold a valid witness $\mathbf{w}$, by letting $\mathrm{V}$ check that the MPC protocol has been correctly carried out "in P's head" on input w. We now consider an MPC protocol $\Pi_{C}$ for the corresponding arithmetic circuit $C$ defined over a finite field $\mathbb{F}$, where the statement information $\mathbf{x}$ (e.g., the plaintext-ciphertext pair) is hard-coded such that $C(\cdot)=f_{\mathbf{x}}(\cdot)$. We assume that the witness is expressed by an $n$-dimensional vector and $C$ takes a set of $n$ input wires denoted 
by $\mathbf{I N}$. We write $\mathbf{w}=(w)_{w \in \mathbb{N}} \in \mathbb{F}^{n}$ for the complete input. ${ }^{2}$ To initialize the protocol, the prover $\mathbf{P}$ first additively secret shares each input $w$ such that $w=w_{1}+\ldots+w_{N}$ in $\mathbb{F}$, and considers each share $w_{i}$ as a private input to a party $P_{i}$. Then $\mathrm{P}$ internally runs $\Pi_{C}$ to obtain view $1, \ldots$, view $_{N}$, where view ${ }_{i}$ consists of $P_{i}$ 's private input $w_{i}$, the random tape of $P_{i}$ and all incoming messages that $P_{i}$ observes during the execution of $\Pi_{C}$. The proof system now proceeds by following the typical "commit-challenge-response" flow. Using a secure commitment scheme, $\mathrm{P}$ sends Commit(view ${ }_{i}$ ) for all $i \in[N]$ as the first message. Upon receiving distinct challenges $i_{1}, \ldots, i_{t} \in[N]$ from the verifier $\mathrm{V}$, the prover $\mathrm{P}$ sends back the corresponding $t$ views view $i_{i_{1}}, \ldots$, view $_{i_{t}}$ as well as the commitment opening information as a response. Finally, the verifier $\mathrm{V}$ accepts the proof iff the opened views are consistent with each other and they produce 1 as output of the protocol $\Pi_{C}$. The (honest verifier) zero knowledge is guaranteed as long as the underlying MPC $\Pi_{C}$ has $t$-privacy in the semi-honest model (i.e., the distribution of any $t$ of fewer views during an honest execution of the protocol is polynomial-time simulatable, given the output from $\Pi_{C}$ and the private inputs of the corresponding parties).

MPC in the preprocessing model. Following [GMO16, $\left.\mathrm{CDG}^{+} 17\right]$, Katz, Kolesnikov, and Wang [KKW18] showed that a particular communication-efficient MPC protocol in the preprocessing model is well suited to MPCitH proofs, and variants of their protocol appear in subsequent work [BN20, Beu20, dDOS19, KZ20]. The core idea of MPC in the preprocessing model is to split the protocol $\Pi_{C}$ into an offline phase $\Pi_{C}^{\text {off }}$ and an online phase $\Pi_{C}^{\text {on }}$. Importantly, the offline phase $\Pi_{C}^{\text {off }}$ can be computed independently of the witness. By precomputing correlated randomness in advance during $\Pi_{C}^{\text {off }}$, one can reduce communication in $\Pi_{C}^{\text {on }}$ drastically. In the traditional MPC setting, this was already used, e.g., in SPDZ [DPSZ12], MiniMAC [DZ13], and TinyOT [NNOB12]. While the original KKW proof system is focused on boolean circuits, it also works with arithmetic circuits in a straightforward manner as observed in [dDOS19, BN20], so we present the latter case here for the sake of generality.

(Offline Phase) The offline phase $\Pi_{C}^{\text {off }}$ of KKW works as follows: for each input wire $w \in \mathbb{I N}$ to the circuit $C$, and for each output wire $z$ from all the multiplication gates, each party $P_{i}$ locally generates random shares $\lambda_{i}^{w}, \lambda_{i}^{z} \in \mathbb{F}$ using its own random tape. Then the parties compute random shares for all internal wires, by running the circuit:

- for each addition gate that takes wires $x$ and $y$ as input, party $P_{i}$ locally computes a new share $\lambda_{i}^{z}=\lambda_{i}^{x}+\lambda_{i}^{y}$ for the output wire $z$;

- for each multiplication gate that takes wires $x$ and $y$ as input, party $P_{i}$ obtains shares of the multiplication triples (sometimes called Beaver triples [Bea92]) $\left(\lambda_{i}^{x}, \lambda_{i}^{y}, \lambda_{i}^{x y}\right)$, such that $\lambda^{x y}=\lambda^{x} \lambda^{y}$.

(Multiplication Triples) To generate multiplication triples in the MPCitH setting, the parties choose $\lambda^{x}$ and $\lambda^{y}$ implicitly by reading their shares from their random tapes. Then to obtain shares of $\lambda^{x y}$, the first $N-1$ parties read random shares from their random tapes. As the prover $\mathrm{P}$ knows all the shares, $\mathrm{P}$ can simply solve for the $N$-th party's share so that the shares reconstruct $\lambda^{x y}$, as required. We call the sequence of values $\lambda_{N}^{x y}$ for all multiplication gates auxiliary information, denoted aux $\in \mathbb{F}^{|C|}$. Note that the complete information needed for the first $N-1$ parties can be derived from their respective seeds seed ${ }_{i}$ used to generate $P_{i}$ 's tape. The information needed for party $P_{N}$ can be derived from $\operatorname{seed}_{N}$ and from aux. Hence, we define each party $P_{i}$ 's state information as follows: for all $i=1, \ldots N-1$, let state $_{i}:=\operatorname{seed}_{i}$, and for $P_{N}$ we have state $_{N}:=\operatorname{seed}_{N} \|$ aux.

(Online Phase) Given the preprocessed state information, the online phase $\Pi_{C}^{\text {on }}$

\footnotetext{
${ }^{2}$ Note that we're slightly abusing the notation here. Throughout, we use the same notations (typically $w, x, y$ and $z$ ) for both wires and wire values, but it should be clear from the context which they indicate.
} 
proceeds by computing the masked witness $\hat{w}=w+\sum_{i \in[N]} \lambda_{i}^{w}$ for each input wire. Now, each gate takes (masked) inputs $\hat{x}=x+\sum_{i \in[N]} \lambda_{i}^{x}$ and $\hat{y}=y+\sum_{i \in[N]} \lambda_{i}^{y}$ and can be computed as follows, where all computations on shares are carried out in $\mathbb{F}$ :

- Addition: each $P_{i}$ locally computes $\hat{x}+\hat{y}$.

- Addition by constant $c$ : each $P_{i}$ locally computes $\hat{x}+c$.

- Multiplication by constant $c$ : each $P_{i}$ locally computes $c \cdot \hat{x}$.

- Multiplication: this computation consumes the triple $\left(\left(\lambda_{i}^{x}\right)_{i \in[N]},\left(\lambda_{i}^{y}\right)_{i \in[N]}\right.$, $\left.\left(\lambda_{i}^{x y}\right)_{i \in[N]}\right)$. Each party $P_{i}$ first locally computes $s_{i}=\lambda_{i}^{z}-\hat{x} \cdot \lambda_{i}^{y}-\hat{y} \cdot \lambda_{i}^{x}-\lambda_{i}^{x y}$ and broadcasts $s_{i}$. Then the masked output $\hat{z}=x y+\sum_{i \in[N]} \lambda_{i}^{z}$ can be obtained as $\hat{z}=\sum_{i \in[N]} s_{i}+\hat{x} \hat{y}$ by each party.

Notice that $\Pi_{C}^{\text {on }}$ only broadcasts once for each multiplication gate, thanks to the correlated randomness computed during the offline phase. All other operations are computed locally by the parties.

\subsection{Picnic}

The signature scheme Picnic is an instance of the MPCitH paradigm described above. The function $f$ is the LowMC block cipher, the signer's secret key is the witness $\mathbf{w}$, and the public key is the statement $\mathbf{x}=(p, c)$. A signature consists of a proof of knowledge of $\mathbf{w}$ such that $f_{p}(\mathbf{w})=c$. In the block cipher notation, if the secret key is denoted $s k$, then the public key is a plaintext-ciphertext pair $(p, \operatorname{LowMC} s k(p))$ where $p$ is a randomly chosen plaintext block, and the signature proves knowledge of a key relating the plaintext $p$ and the ciphertext $\operatorname{LowMC}_{s k}(p)$. The proof is made non-interactive by the Fiat-Shamir transform, and the message to be signed is bound to the proof by hashing it into the challenge.

The Picnic specification [Pic20] and NIST submission includes parameter sets using both the $\mathrm{ZKB}++$ proof system and the KKW system, as well as specific choices of parameters for LowMC. Since the KKW-based parameters (referred to as Picnic3) are the most efficient in terms of signature size, we choose to focus on those in this paper. In particular our masked implementation is limited to the parameter set Picnic3-L1. Fig. 6 describes the KKW proof system at a high level, and Algorithm 14 describes Picnic3 signing in full detail.

\subsection{Side-Channel Attacks and Threat Model}

Physical attacks are a threat for cryptographic implementations. Attacks such as sidechannel analysis (SCA) can be used to extract secret keys by observing physical properties of an implementation, such as timing, power consumption or electromagnetic emanation [Koc96, KJJ99, QS11]. A popular countermeasure to SCA is known as masking [CJRR99a, ISW03]. Masking can protect against a broad class of SCA and probing attacks by splitting secrets into independent shares. A popular model for analyzing masking schemes is the t-probing model, where the adversary can probe one (or more) shares of the masked variable [ISW03]. Briefly, a probing adversary may invoke a cryptographic implementation multiple times with chosen inputs. Before each call, the adversary can choose a set of up to $t$ wires of the circuit and observe the values on these wires during the invocation. After $c$ calls, an attacker can then combine the $c \times t$ observations in arbitrary ways to extract information about sensitive variables. This model is closely related to concrete physical attacks: For example, in $\left[\mathrm{KGM}^{+} 20\right]$, eight simultaneous probes are given as an upper limit achievable by modern commercially-available probe stations, and we therefore assume that $t$ is at most sixteen. While the $t$-probing model is a clean theoretical model, Duc et al. [DDF19] showed that security in this model implies security in the more practical SCA-inspired noisy leakage model [PR13]. To protect against multi-probe 
attacks, generally higher-order masking is applied, where the number of independent shares is increased. Higher-order SCA is expensive, as the number of measurements needed grows exponentially with the masking order, effectively limiting the attack order or the number of simultaneous probes an adversary may use (which we assume is below sixteen). Kranchenfels et al. $\left[\mathrm{KGM}^{+} 20\right]$ describes a new attack technique, laser logic state imaging, that can potentially have an unlimited number of probes, however this attack is quite new and may not be widely applicable, but in any case must be mitigated with countermeasures below the software level (at the package, device or circuit level). Besides probing and SCA, there are further physical attacks like fault analysis [BDL97] that we do not address in this work.

In this work, we use the noisy leakage model introduced by Chari et al. [CJRR99b] and extended by Prouff and Rivain [PR13]. The model enables an adversary to obtain each intermediate value perturbed with a noisy leakage function. Furthermore, as stated above we use the connection between probing and the noisy leakage model given by Duc et al. [DDF19]. Therefore in our threat model, a probing adversary reflects the capabilities of a real world adversary such as DPA. We assume an adversary who can access the physical device that can run the Picnic3 signature scheme. They can measure side-channel traces, such as power or electromagnetic emanation, of the device while signing chosen messages. Moreover they obtain the signatures as output, and can verify (and thus see the revealed values) or use them arbitrarily in an attack. Observe that according to the noisy leakage model the side-channel trace contains each intermediate value perturbed with a noisy leakage function. Depending on the signature the revealed values vary and the adversary can employ these variables, to recover the secret.

\subsection{Security Notions for Masking Countermeasures}

For more comprehensive background we refer readers to Appendix G. In the following, we fix a finite field $\mathbb{F}$. As explained above, we are working in the $t$-probing model which allows an attacker to obtain the value of $t$ variables per run of the primitive. The most common technique to mitigate side-channel attacks is by encoding sensitive variables via an additive (or polynomial-based) secret sharing into $T>t$ parts. We use the additive approach, and say that a vector $\left(v_{j}\right)_{j \in[T]} \in \mathbb{F}^{T}$ is a T-encoding of $v:=\sum_{j \in[T]} v_{j}$. For readability, we often write $\langle v\rangle$ instead of $\left(v_{j}\right)_{j \in[T]}$. For a subset $I \subseteq[T]$, let $\langle x\rangle_{I}=\left(x_{i}\right)_{i \in I}$ and furthermore $\bar{I}=[T] \backslash I$. Variables are shared both to protect against side-channel attacks and as part of the MPC protocol. To distinguish between these situations, we call a sharing between parties in the MPC protocol a sharing and use the term encoding when the goal is to protect against side-channel attacks. In this work, we aim to prove that our basic building blocks meet the following standard security notions $\left[\mathrm{BBD}^{+} 16\right]$.

Definition 1 ( $t$-NI, $t$-SNI). Let $G$ be a gadget with inputs in $\mathbb{F}^{T}$ and $t<T$. Suppose that for any set of $t_{1}$ intermediate variables and any subset of $O$ of output indices with $t_{1}+|O| \leq t$, there exists a subset of indices $I$ such that the output distribution of the $t_{1}$ intermediate variables and the output variables $y_{\mid O}$ is perfectly simulatable from $I$. Then

(i) if $|I| \leq t_{1}+|O|$ we say $G$ is $t$-non-interfering ( $t$-NI), and

(ii) if $|I| \leq t_{1}$ we say $G$ is $t$-strong-non-interfering ( $t$-SNI).

The above definition of SNI as well as its composability result can be generalized for a gadget with multiple input/output encodings [CS20]. We also say $G$ is $t$-SNI with uniform output-distribution, if the outputs of $G$ which are not affected by any probes are uniformly distributed (see Appendix G for a definition). 


\section{Probing Attacks on Picnic3}

We describe two probing side-channel attacks against Picnic3 and experimentally confirm them against our M4 port of the optimized Picnic3 implementation. The second attack is deferred to Appendix A since it is a direct consequence of previous work [GSE20]. Probing attacks usually exploit weak leakage of intermediate variables, gathered from several measurements. As described in [SBWE20] and in [GSE20], the values revealed by the prover to allow the verifier to check the consistency of the MPC protocol can be employed by an adversary in a side-channel attack. We assume the same scenario. Furthermore, we assume a leakage model where an implementation leaks weak and noisy information about each intermediate variable, therefore measurements of the MPC-in-thehead simulation have a weak and noisy dependence on secret values due to the revealed values. As mentioned above, we make use of the RvR tests to show the clear presence of leakage.

\subsection{Probing the Unopened Online Phase}

This attack is novel, as it is specific to MPCitH with preprocessing, and only occurs when 5 protocol rounds are compressed to 3 . Hence the attack below works in principle for any direct implementation of signatures derived from three-round $\mathrm{KKW}$-based protocols. We also remark that this attack cannot be mitigated by the SNIitH approach [SBWE20]; in particular, the attack below works independently of the number of unopened parties' views since it targets an input to the MPC (i.e., $\hat{w}$ ), not a share of the secret. We are thus motivated to design an alternative solution to thwart this attack in the next section.

We first note the three-round KKW scheme executes both the offline and online phase of each MPC instance, in contrast to the five-round case. We denote by $\mathcal{C}$ the executions chosen for the online phase, i. e., the executions where the offline phase is not public.

The attack exploits the following: if the $k$-th execution of the offline phase is selected to be part of the signature (i.e., if $k \notin \mathcal{C}$ ), the preprocessed masks and state of all $N$ parties is made public for the verifier, therefore the corresponding online phase must remain hidden. Concretely, since the secret witness wire value $w$ is masked by random bits in step 1c of the prover in Fig. 6, the attacker's goal is to learn the masked witness wire values $\hat{w}^{(k)}$ in execution $k$ for the unopened online executions $k \notin \mathcal{C}$. Since $\hat{w}^{(k)}=\lambda_{1}^{w,(k)}+\ldots+\lambda_{N}^{w,(k)}+w$ and $\lambda_{i}^{w,(k)}$ is made public (for all $i$ ), by probing $\hat{w}^{(k)}$ the attacker can solve for the secret key bit $w$. Here, $\lambda_{i}^{w,(k)}$ denotes the value of $\lambda_{i}^{w}$ in execution $k$.

In order to validate the attack we use our experimental setup (as described in Section 6) and the RvR approach. The experiment shows that there is an exploitable leakage, i.e., an amount of leakage sufficient, despite measurement noise, to allow recovery of intermediate values that depend on the secret key. For this experiment, we reduce the Picnic3 parameters (as in Fig. 6) $M$ and $\tau$ to 4 and 2 respectively, in order to collect traces more quickly, however we keep the number of parties $N$ as 16 and collected traces corresponding to execution of the first MPC instance. During the collection phase, we run the Picnic3 signing function with random messages and a fixed secret key. More specifically, we measure the execution of the first line of Algorithm 18, and collect 22,056 side-channel traces. Note that the root seed is also random due to the choice of a random message to be signed. We first separate the traces belonging to signatures that reveal the first preprocessing phase, since our measurement covers the first MPC instance. The reduced number of MPC instances is only to reduce the number of possible challenges and to increase the number of traces per challenge. Then we classify the remaining traces into two sets according to the revealed values $\lambda_{1}^{w,(k)}+\cdots+\lambda_{16}^{w,(k)}$. The result of the analysis in Fig. 1 (left side) shows a clear dependence between the unrevealed value $\hat{w}^{(k)}$ and the observable trace, as the $|t|$-value exceeds 5.7 which indicates an exploitable leakage. As seen in the right hand side of the Fig. 1, the leakage becomes clear after 2,725 traces. 

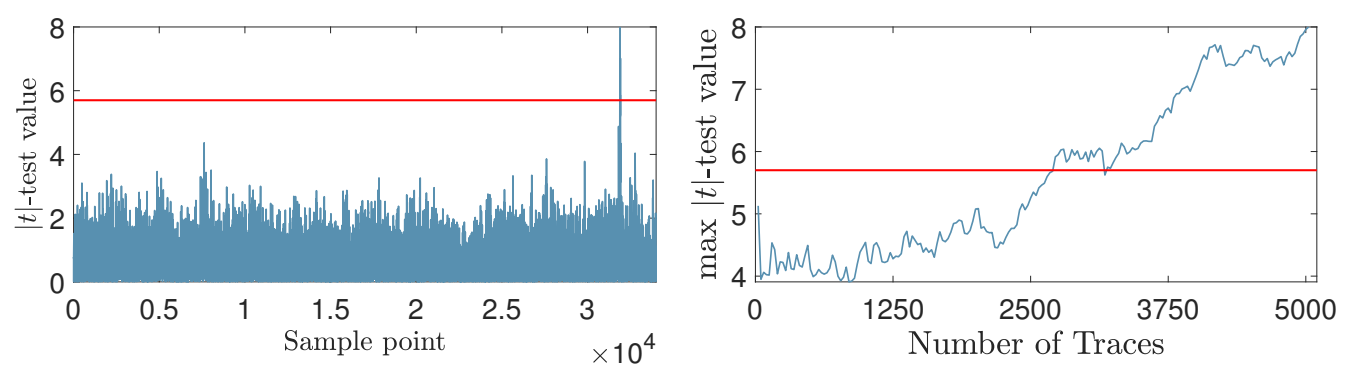

Figure 1: A first-order RvR test on the unprotected Picnic3 implementation using revealed values from the offline phase. The traces are classified based on the bit $\lambda_{1}^{w}+\cdots+\lambda_{16}^{w}$. Values above the 5.7 threshold (red line) indicate strong leakage (left). Moreover, the maximum $|t|$-value increases with respect to the number of traces and the leakage becomes clear after 2,725 traces (right).

The code we measure (the first line of Algorithm 18) corresponds to the calculation of roundkey $y_{0}$ thus the leakage corresponds to the bits of roundkey ${ }_{0}$ which is equal to $\operatorname{mat} \operatorname{Mul}\left(\hat{s k}, K_{0}\right)$. Solving the equation for the $s k=\hat{s k}-\left(\lambda_{1}^{s k}+\cdots+\lambda_{16}^{s k}\right) K_{0}$ where $\left(\lambda_{i}^{s k}\right.$ is known for all $i$ and $K_{0}$ is a constant) leads to the secret value $s k$.

\section{Masking Three-Round KKW}

In this section we present our masked proof system following the three-round KKW protocol. We first strive for a provably NI-secure algorithm without specifying any particular circuit. In Section 5 we describe more concrete operations tailored to the LowMC circuit of Picnic3, and optimize by partially unmasking several hash computations (and discuss the security implications). The circuit in this presentation is a generic circuit $C$ such that $C\left((w)_{w \in \mathbb{I N}}\right)=1$, where each $w$ is seen as an input wire value to the circuit.

In the description below, we additively secret share some variables in two dimensions: a share held by each party (indexed by $i \in[N]$ ), further shared $T$ times within each party (indexed by $j \in[T]$ ). For example, $\lambda_{i}^{x}$ denotes a share of of $\lambda^{x}$ held by the $i$-th party such that $\lambda^{x}=\sum_{i \in[N]} \lambda_{i}^{x}$; the value $\lambda_{i, j}^{x}$ for $j \in[T]$ denote shares such that $\lambda_{i}^{x}=\sum_{j \in[T]} \lambda_{i, j}^{x}$. The shares $\lambda_{i}^{x}$ are as in the KKW protocol, and the extra $T$-wise encoding of this value is required for SNI security. Note that we only apply the notation $\langle\cdot\rangle$ (see Section 2.4) on the $T$-wise encoding required for the masking countermeasure and never for the sharing of the KKW protocol. The functions requiring $T$-wise masked computation are marked in orange (e.g., $\langle y\rangle \leftarrow \mathrm{H}(\langle x\rangle)$ indicates that a hash function $\mathrm{H}$ is masked). Most of the randomness used in the protocol is from the random tapes of the parties; this randomness is derived from a seed, so that part of it may be efficiently communicated to the verifier (by sending the seed). Some of the masked operations will require additional randomness (e.g., to refresh a secret encoding), and this is sampled from the platform random number generator $(\mathrm{RNG})$, since it is only required by the signer.

\subsection{Masked Operations}

We present our masked version of the KKW prover in Fig. 2. The function masked_offline in Algorithm 1 computes the offline phase $\Pi_{C}^{\text {off }}$ from Section 2.1 in a straightforward way. The function masked_online in Algorithm 2 corresponds to a masked version of $\Pi_{C}^{\text {on }}$. The SNI-secure multiplier SMul( ) is defined in Algorithm 11. Note that SMul( ) is $t$-SNI with uniform output-distribution, as evident from the proof that it is $t$-SNI $\left[\mathrm{BBD}^{+} 16\right.$, Proposition $2]$. For ADD gates, the only change is to work on $T$-encodings $\langle\hat{x}\rangle,\langle\hat{y}\rangle$, rather than on $\hat{x}, \hat{y}$ directly. Interestingly, the MUL gates can also be computed with a straight-forward 


\section{Algorithm Masked KKW Prover}

Inputs The prover holds a circuit $C$ as a statement and an encoded witness $\langle\mathbf{w}\rangle=\{\langle w\rangle\}_{w \in I N}$ such that $C(\mathbf{w})=1$. Values $M, N, \tau$ are parameters of the protocol.

Commit For each $k \in[M]$, the prover does:

1. Choose uniform $\left\langle\operatorname{seed}^{(k)}\right\rangle$ and use to generate values $\left\langle\operatorname{seed}_{1}^{(k)}\right\rangle, \ldots,\left\langle\operatorname{seed}_{N}^{(k)}\right\rangle$. Also the prover computes $\left\langle\operatorname{aux}^{(k)}\right\rangle \in \mathbb{F}^{|C|}$ as in Algorithm 1. For all $i=1, \ldots N-1$, let $\left\langle\operatorname{state}_{i}^{(k)}\right\rangle=\left\langle\operatorname{seed}_{i}^{(k)}\right\rangle$ and let $\left\langle\right.$ state $\left._{N}^{(k)}\right\rangle=\left\langle\operatorname{seed}_{N}^{(k)}\right\rangle||\left\langle\operatorname{aux}^{(k)}\right\rangle$.

2. Commit to the offline phase:

$$
\begin{aligned}
\left\langle\operatorname{com}_{i}^{(k)}\right\rangle & =\mathrm{H}\left(\left\langle\operatorname{state}_{i}^{(k)}\right\rangle\right) \text { and reconstruct } \operatorname{com}_{i}^{(k)} \text { for all } i \in[N] \\
\text { com_off } & =\mathrm{H}\left(\operatorname{com}_{1}^{(k)}, \ldots, \operatorname{com}_{N}^{(k)}\right) .
\end{aligned}
$$

3. Compute encodings of masked witness $\left\langle\hat{w}^{(k)}\right\rangle=\left\langle\lambda_{1}^{w}\right\rangle+\ldots+\left\langle\lambda_{N}^{w}\right\rangle+\langle w\rangle$ for each $w \in$ IN, where $\left\langle\lambda_{i}^{w}\right\rangle$ is the randomness used to mask the witness and is read from the random tape defined by $\left\langle\right.$ state $\left._{i}^{(k)}\right\rangle$.

4. Simulate the online phase of the $N$-party protocol as in Algorithm 2 and produce $\left\langle\operatorname{msgs}_{1}^{(k)}\right\rangle, \ldots,\left\langle\operatorname{msgs}_{N}^{(k)}\right\rangle$.

5. Commit to the online phase:

$$
\left\langle\text { com_on }^{(k)}\right\rangle=\mathrm{H}\left(\left\{\left\langle\hat{w}^{(k)}\right\rangle\right\}_{w \in \mathbb{N}},\left\langle\operatorname{msgs}_{1}^{(k)}\right\rangle, \ldots,\left\langle\operatorname{msgs}_{N}^{(k)}\right\rangle\right)
$$

and reconstruct com_on ${ }^{(k)}$.

6. The prover refreshes the encoding of witness $\langle w\rangle=\operatorname{RefreshM}(\langle w\rangle)$ for each $w \in \operatorname{IN}$.

Compute $h_{\text {off }}=\mathrm{H}\left(\right.$ com_off $^{(1)}, \ldots$, com_off $\left.^{(M)}\right)$ and $h_{\text {on }}=\mathrm{H}\left(\right.$ com_on $^{(1)}, \ldots$, com_on $\left.^{(M)}\right)$ and send $h^{*}=\mathrm{H}\left(h_{\text {off }}, h_{\text {on }}\right)$ to the verifier.

Challenge The prover receives the following challenges from the verifier: a uniform $\tau$-sized set $\mathcal{C} \subset[M]$ and $\mathcal{P}=\left\{i_{k}\right\}_{k \in \mathcal{C}}$ where each $i_{k} \in[N]$ is uniform.

Response For each $k \in[M] \backslash \mathcal{C}$, the prover sends reconstructed seed ${ }^{(k)}$ and com_on ${ }^{(k)}$ for all to the verifier. For each $k \in \mathcal{C}$, the prover sends reconstructed values $\operatorname{com}_{i_{k}}^{(k)},\left\{\text { state }_{i}^{(k)}\right\}_{i \neq i_{k}},\left\{\hat{w}^{(k)}\right\}_{w \in \mathbb{I N}}$ and $\operatorname{msgs}_{i_{k}}^{(k)}$ to the verifier.

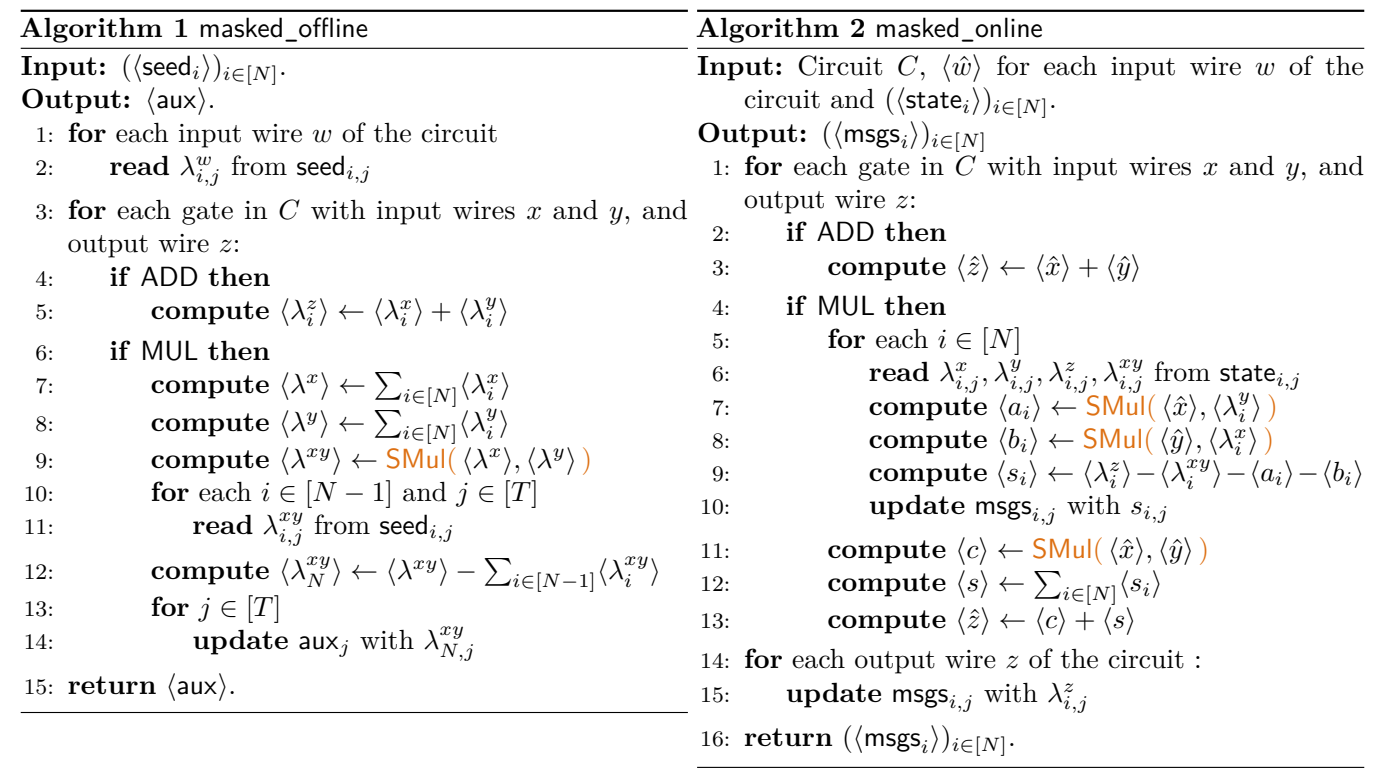

Figure 2: Our masked version of 3-round KKW prover. 
adaptation by also encoding the masks $\lambda^{x}, \lambda^{y}, \lambda^{z}$, and $\lambda^{x y}$. Recall that $\hat{x}=x+\lambda^{x}$, and $\lambda^{x}$ is shared among the parties, where party $i$ has share $\lambda_{i}^{x}$ (resp. $\lambda_{i}^{y}, \lambda_{i}^{z}$, and $\lambda_{i}^{x y}$ ). Each party thus stores their share $\lambda_{i}^{x}$ as a $T$-encoding $\left\langle\lambda_{i}^{x}\right\rangle$ (resp. $\left\langle\lambda_{i}^{y}\right\rangle,\left\langle\lambda_{i}^{z}\right\rangle$, and $\left\langle\lambda_{i}^{x y}\right\rangle$ ). Each party's broadcast value $s_{i}$ will now also consist of the $T$-encoding $\left\langle s_{i}\right\rangle$ as follows:

$$
\left\langle s_{i}\right\rangle=\left\langle\lambda_{i}^{z}\right\rangle-\left\langle\lambda_{i}^{x y}\right\rangle-\operatorname{SMul}\left(\langle\hat{x}\rangle,\left\langle\lambda_{i}^{y}\right\rangle\right)-\operatorname{SMul}\left(\langle\hat{y}\rangle,\left\langle\lambda_{i}^{x}\right\rangle\right) .
$$

\subsection{Security Analysis}

We employ the definition of non-interference by $\left[\mathrm{BBD}^{+} 16\right]$ which guarantees security against $t$ probes for $t<T$ as proposed by Ishai et al. [ISW03]. Recall that a probing adversary may invoke a cryptographic implementation multiple times with chosen inputs and before each call, can fix an arbitrary set of up to $t$ wires of the circuit and observe the values during the invocation. We use a more polished security notion known as $t$-non-interference $(t$-NI) and $t$-strong non-interference $(t$-SNI) defined in Appendix G.

In the security analysis we focus on a single MUL operation as extracted from $\mathrm{Al}$ gorithms 1 and 2, denoted KKW_MUL, presented as Algorithm 22 and 23, as the ADD operation is linear and thus trivially NI. See Appendix H for the proofs of the lemmas.

Lemma 1. Let $G$ be the KKW_MUL gadget as described in Algorithm 22. Then, $G$ is $t$-SNI for all $t<T$, if SMul( ) is t-SNI with uniform output-distribution.

Lemma 2. Let $G$ be the KKW_MUL gadget as described in Algorithm 23. Then, $G$ is $t$-SNI for all $t<T$, if SMul( ) is $t$-SNI with uniform output-distribution.

To further support our security analysis, we utilized maskVerif $\left[\mathrm{BBC}^{+} 19\right]$ to confirm that both KKW_MUL offline and online gadgets are SNI-secure up to the order 4. Although we further provide the verification scripts for the orders 5,6 and 7 , we did not run these, due to higher combinatorial complexity of maskVerif. However, our manual security analysis indeed guarantees SNI security of higher-order masking.

As we have shown that all components of Algorithm 1 and Algorithm 2 are SNI, the composability guaranteed by Lemma 3 as well as its generalization to multi-input/output SNI gadgets [CS20] implies the following theorem by adding suitable refresh gadgets (depending on the topology of circuit $C$ that KKW is instantiated with). Note that SNI security of the refresh gadget (recalled in Algorithm 12) is already proved by Barthe et al. $\left[\mathrm{BBD}^{+} 16\right]$

Theorem 1. Suppose $\mathrm{H}(\mathrm{)})$ and RefreshM( ) are $t$-SNI secure gadgets for all $t<T$. The masked version of $\mathrm{KKW}$ presented in Fig. 2 is $t$-NIo for all $t<T$ and for public outputs $\left\{\operatorname{com}_{i \neq i_{k}}^{(k)}\right\}_{k \in \mathcal{C}},\left\{\operatorname{com}_{i}^{(k)}\right\}_{k \in[M] \backslash \mathcal{C}, i \in[N]},\left\{\operatorname{com}_{-} \text {off }{ }^{(k)}\right\}_{k \in[M]}$ and $\left\{\operatorname{com}_{-} \text {on }{ }^{(k)}\right\}_{k \in \mathcal{C}}$.

The public outputs stated above are not explicitly included in the KKW proof. We thus have to validate the security of proof system in case these values are made public, which is straightforward since they are recomputed as part of verification from the other information in the proof (see the verification step of Fig. 6). Furthermore, note that masking the hash function $\mathrm{H}(\mathrm{)})$ is important. There are scenarios where the inputs to $\mathrm{H}(\mathrm{)}$ are sensitive, but the outputs are not (such as step 5 in Fig. 2). Leaving the hash function unmasked might therefore leak information about these sensitive inputs.

\section{Masking Picnic}

We start by analyzing the hashing operations in signature generation to determine which ones must be masked, then discuss the options for masking SHA3/SHAKE, and introduce the half-masking technique, then estimate the overhead of masking the hash invocations. Our masked implementation of the Picnic3 signature generation function is a rather direct 
adaptation of the masked KKW proof protocol from Section 4. When compared to Section 4, the circuit is LowMC, and operations are done on $N$-bit words packed with a secret share from each of the $N$ parties. Fig. 7 (in Appendix C) gives an overview of the protections for each hashing operation in signature generation. A complete specification of our protected implementation, mirroring the official Picnic specification [Pic20], is given in Appendix C.

\subsection{Implementation Security Options}

We implemented several versions of masked Picnic3, of which we highlight two: (1) a provably NIo-secure implementation (as a direct consequence of Theorem 1) and (2) a performance-oriented implementation with partially unmasked non-sensitive intermediate values. For the former, we inserted the share refresh gadget (RefreshM) according to the generic composition rule stated in Lemma 3, and we verified with maskVerif that our complete specification of fully masked Picnic3 (see Appendix C.1) is indeed NIo-secure. On the other hand, we do not claim that our second implementation is NIo-secure, as there are some gaps between the analysis of Section 4 and this implementation, that we consciously allowed, in order to improve performance. We now explain these gaps and argue that they do not impact practical security, and in Section 6 we confirm the absence of leakage experimentally.

First, according to Algorithm 4, all intermediate seeds must be $T$-encoded until they are reconstructed at lines 29 and 32 and, as we argue in Section 5.2, reading $t$ bits of a seed reduces security by at most $t$ bits. As we assume $t$ to be small, we accepted this risk to reduce the cost of masking SHAKE and the memory required to store seeds.

By selectively masking the hash function calls (as described in Section 5.2) as opposed to masking all hash function calls, up to $t$ bits of a single-use seed may leak to a side-channel attacker capable of accurately reading $t$ bits from a single trace. (Since the seed is only ever used once, and the signature is randomized, subsequent traces have a fresh seed.) Against such an attack, the security of our L1 implementation decreases from 128 to $128-t$ bits against side-channel attacks, and we assume that $t \leq 16$ in Section 2.3. As shown in Sections 5.4 and 6 this optimization gives significant performance gains, so we see this as a reasonable trade-off. Recent work by Kannwischer et al. [KPP20] describes single-trace attacks on the unprotected XKCP Keccak implementation. These attacks use a single trace recorded during the computation of $y=\operatorname{SHAKE}(s k \| x)$ and aim to recover all of a secret key $s k$, or part of $y$. While single-trace attacks could threaten some of the unprotected hash calls in our optimized implementation (e.g., when deriving the per-party or per-MPC instance seeds), the results of [KPP20] do not extend to the M4, and the length constraints on $s k, x$, and $y$ in our application. Future work may improve single-trace attacks, and in that case the conclusion of [KPP20] is that lightweight countermeasures will provide effective mitigation.

Half-masking (discussed in Section 5.3) also introduces the assumption that KangarooTwelve $\left[\mathrm{VWA}^{+} 21\right]$ is a secure hash function. This assumption is only for security against the type of $t$-probing side-channel attack we consider; and half-masking can be used by individual implementations without changes to the Picnic specification. We provide benchmarks in Section 6 showing the performance advantage of half-masking: based on this and the fact that security of KangarooTwelve appears to be a relatively mild assumption, we enable half-masking by default in our implementation.

Finally, our provable security analysis assumed an SNI-secure hash implementation. Although one could use the fully SNI-secure masked Keccak as suggested by Barthe et al. $\left[\mathrm{BBD}^{+} 16\right]$, other previous works [BDPA10, Dae17, GSM17] achieved more efficient implementations with smaller amounts of random bits, albeit without a provable security guarantee. We implement three instances of masked Keccak (named IND, DOM, and SNI) with different security levels, which we explain in detail in Section 5.3. In Section 6, we compare the concrete performance and perform practical leakage analysis. From these experiments, we conclude that our implementation of IND - the fastest instance among three - does not leak information. 


\subsection{Side-Channel Protections for Hashing in Picnic}

Hashing Operations for Signing The concrete sign operations are described in Algorithm 3, following the Picnic specification [Pic20, Section 7.1]. When compared to the stylized description of KKW in Fig. 6, here we include more details and list all hashing operations, since we will analyze them with respect to the probing attacks below. Some of the functions related to expanding seeds using a tree construction or creating a Merkle tree of commitments (gen_seed, get_leaves, build_tree, and open_tree) are left to the specification for simplicity.

The hash function calls are denoted by $\mathrm{H}$ and we omit the byte used for domain separation present in the specification. The KDF expands an arbitrary length input to an arbitrary length output. Both $\mathrm{H}$ and KDF are instantiated with the SHAKE XOF.

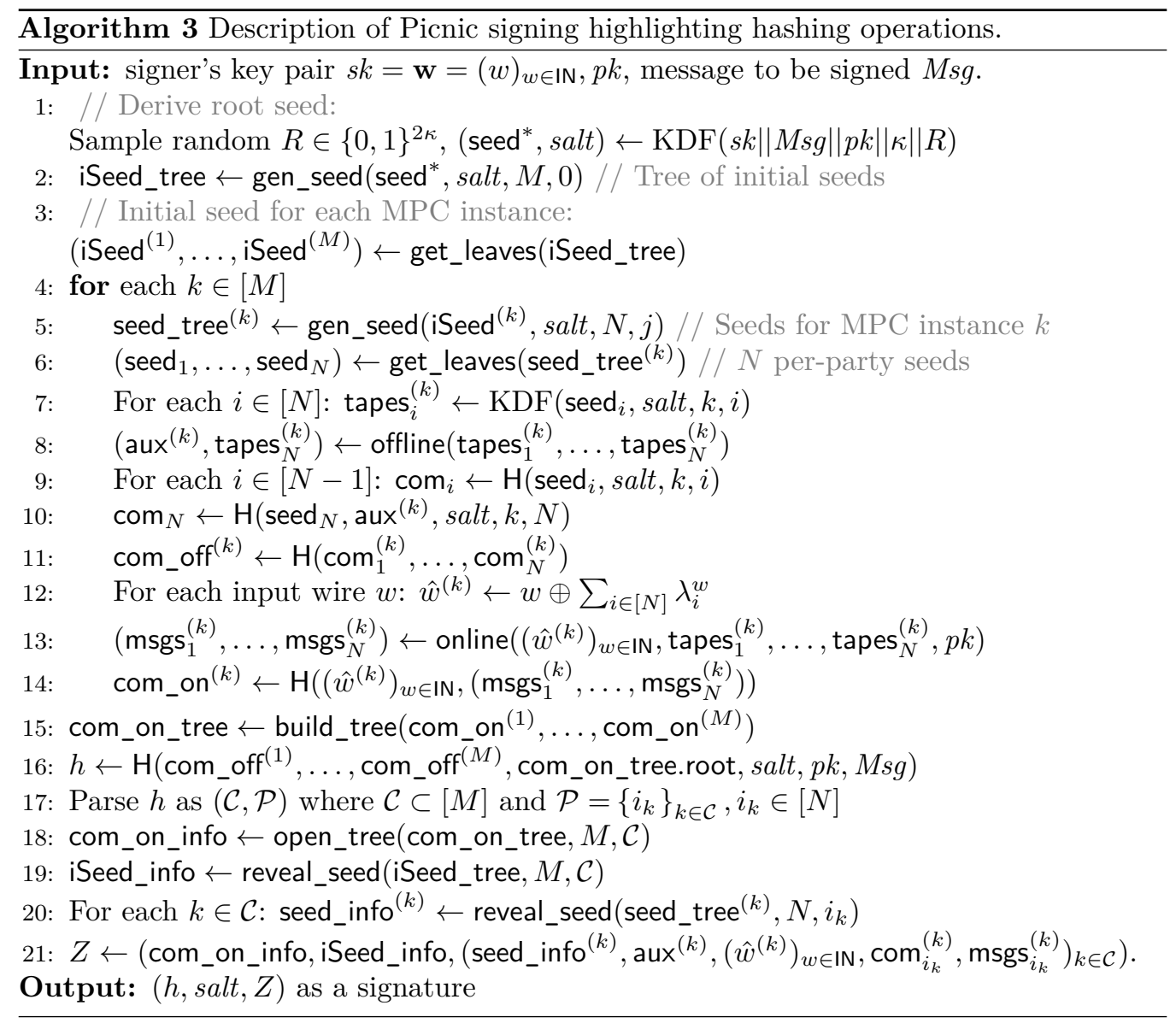

We now consider which hashing operations must be protected against side-channel attacks, and to what degree. The Picnic specification supports randomized signatures (and recommends this option, following [AOTZ20]) by appending a random value to the KDF input when deriving the root seed. We assume this option is used throughout, as otherwise the cost of side-channel protections would be significantly higher, since all hash function calls would require masking (as opposed to only $35 \%$ shown below), and all random seeds would need to be $T$-encoded. First, we note that all inputs to the challenge computation are public, so this hash does not need to be masked. We now analyze the other hash function calls in order. Fig. 7 (in Appendix C) gives an overview of the operations. When referring to individual hashing operations, we refer to the steps in Algorithm 4. 
Deriving the root seed. For step 1, the SHAKE XOF is used as a KDF to derive a root seed for signature generation. The input $s k$ must be protected from side-channel attacks. As a first option, one could choose the root seed at random, and avoid the KDF altogether. However, deriving the root seed from the secret key and random data hedges against failures in the RNG, see the analysis for Picnic in [AOTZ20]. If $s k$ is stored $T$-encoded, then we can hash all of the shares in place of $s k$, and append a random value. Our implementation masks this hash function call since it is relatively cheap in the context of a signature, and it makes testing easier because our implementation can produce signatures that match known test vectors.

Deriving other seeds. When generating the seeds in steps $2,3,5$, and 6 , protecting against the limited type of leakage we consider in this work is not necessary, since seeds are unique per-signature and are always hashed before use. Suppose an attacker $A$ can read $t$ bits of a leaf or intermediate seed $s$. With overwhelming probability each seed is only ever used in one signature, so traces from multiple signing operations will not give more information about $s$.

There are three possible uses of $s$ to consider. When $s$ is a seed from a leaf of the tree, case 1 is that $s$ is hidden and the attacker has a commitment to it (computed in steps 9 and 10), and case 2 is when $s$ is used to seed KDF (in step 7), and $A$ has some of the output bits. In case $3, s$ is a hidden intermediate seed, the attacker has one of the two child seeds, derived by hashing $s$.

We can model all three cases as the attacker having $C=\mathrm{H}(s)$ along with $t$ bits of $s$, where $\mathrm{H}$ is a secure hash function. In practice $\mathrm{H}$ is the SHAKE XOF, which the existing analysis of Picnic already assumes is a random oracle. Then if $A$ makes $q$ queries to $\mathrm{H}$, they recover the missing $\kappa-t$ bits of $s$ with probability not more than $q / 2^{\kappa-t}$. This considers only a single seed and digest, which we can do since each input to $\mathrm{H}$ is unique, by construction (the Picnic spec uses a domain separation value, random salt, and counters to prevent multi-target attacks [DN19]). In practice $\kappa \geq 128$ and $t$ will be 16 or less (see Section 2.3), therefore the security of our implementation is still at least 112 bits.

Computing random tapes. In step 7 we expand the per-party seeds to random tapes. The inputs do not need to be protected (as discussed in the previous paragraph), but all output bits must be protected, since some of the random tape bits will correspond to shares of the unopened party and must be kept secret as shown in in Section 3. We mask these calls, so the output is $T$-encoded (which increases the amount of memory required to store the tapes by a factor of $T$ ).

Computing commitments. In step 9, a commitment of the form $H$ (seed $\|$ salt) is computed. Here the private input is a seed, which is not sensitive to leakage of up to $t$ bits, as discussed above, and the output is public. Therefore, step 9 does not require masking.

In step 10, the last party's commitment has the additional input aux, which is sensitive to leaking of individual bits. We must mask this call, but since the output is public, we can use the half-masking technique of Section 5.3.

In step 11, we hash only public values, and no masking is required. In step 14, all inputs are sensitive to leaking individual bits (e.g., $\hat{w}$ is sensitive due to the attack described in Section 3.1). Because the output is public, the half-masking technique is applicable.

\subsection{Masking SHAKE}

We implement multiple methods to protect the SHA3 family of function against DPA attacks. In all of them, the Keccak- $f$ state array $A$ is secret shared into two arrays $a, b$, such that $A=a+b$. In the basic method proposed in [BDPA10], the linear operations are performed on the individual state arrays, then for the non-linear step (denoted $\chi$ ) $A_{i} \leftarrow A_{i}+\left(A_{i+1}+1\right) A_{i+2}$ the shares $(a, b)$ are updated as $a_{i} \leftarrow a_{i}+\left(a_{i+1}+1\right) a_{i+2}+a_{i+1} b_{i+2}$ and $b_{i} \leftarrow b_{i}+\left(b_{i+1}+1\right) b_{i+2}+b_{i+1} a_{i+2}$, evaluated left-to-right. The cost of the linear 
operations are doubled, addition of constants have the same cost, and the cost of $\chi$ is doubled, plus two additional AND and XOR operations, so the computational cost of the masked round function is roughly doubled. One must also consider the cost of generating random values to create the secret shares. This method (herein called IND) only achieves independence from the native variables, and the same approach can be generalized to three or more shares. In Domain-Oriented Masking (DOM) [GSM17], the AND operations between shares $a$ and $b$ are further protected to satisfy SNI-security with a random mask $Z$ as $\left(a_{i+1} b_{i+2}+Z\right)$ and $\left(b_{i+1} a_{i+2}+Z\right)$, respectively. However, this is still not sufficient for the masked Keccak as a whole to be SNI secure: due to the $\theta$-layer, which applies a linear transform to the state array $A$, both inputs to the AND gadget in $\chi$ depend on the same previous state bit. This is a typical pattern of insecure composition observed in $\left[\mathrm{BBD}^{+} 15 \mathrm{~b}, \S 2.3\right]$. Therefore, the third method (denoted by SNI) achieves SNI-security by additionally refreshing shares of the state array $A$ for every invocation of $\chi$, as already suggested by Barthe et al. $\left[\mathrm{BBD}^{+} 16, \S 8.2\right]$.

Half-Masked SHAKE. When expanding a seed to a random tape, we have shown that security is maintained when leaking a small part of the seed ( $t$ bits of fewer), so the input is not sensitive to this bounded leakage, but the output is sensitive. Conversely, when creating a commitment, the individual input bits may be sensitive but the output is public. An established assumption (for SHAKE128) is that security is preserved using only half the number of rounds, and there is a proposal called KangarooTwelve (K12) [VWA ${ }^{+}$21], that uses 12 instead of 24 rounds. Therefore, for short inputs and outputs, one can view SHAKE as two calls to K12, and mask only one of the calls. In the case of sensitive inputs, we mask the first 12 rounds: an attacker who learns the state at the 13th round is effectively given a K12 digest of the input, which sufficiently hides the input under the assumption that K12 is a secure hash function. Similarly, when only the output bits are sensitive, we mask the last 12 rounds, any state bits observed by the adversary in round 11 does not leak useful information about the output assuming that K12 is secure.

\subsection{Estimated Overhead of Hash Function Masking in Picnic}

Here we provide a rough estimate of the overhead introduced by masking the SHAKE calls in Picnic3, which will have a high impact on the cost of signing since hashing is a large portion of the signing time (e.g., at L1 it is about 57\% of the signing time on x64 [KZ20], and for our ARM M4 implementation it is about $71 \%$ ).

- For seed tree hashing, we have about $M+\log _{2} M$ hashes to compute the round seeds, and $M N+M \log _{2} N$ hashes for the per-party seeds. None of these must be masked.

- For random tape expansion, we have $M N$ hashes, all of which must be masked.

- For commitments, we have $N M+2 M+\log _{2} M$ hashes and must mask $2 M$ of these. The total number of hashes is thus $3 M N+3 M+2 \log _{2} M+\log _{2} N$ and $M N+2 M$ of these must be masked. At L1, all hash operations involve one call to Keccak- $f$, so all calls have approximately the same cost. Again at L1, $M=250, N=16$ so we find that about $35 \%$ of hashing must be masked. Since all masked hash operations have either non-sensitive input or output they need only be half-masked (as explained below).

Now suppose we focus on first-order protection (the case $T=2$ ), and assume that masked SHA-3 is about 2.73 times slower than unmasked SHA-3, and that a half-masked SHA-3 is about 1.95 times slower (these are the ratios from our implementation described in Section 6). Then we expect a $1.61 \mathrm{x}$ increase in time spent hashing in masked Picnic3, and $1.35 \mathrm{x}$ increase when half-masking is used.

\section{Implementation and Experimental Evaluation}

In this section we benchmark our implementation and discuss performance, then describe our experiments to ensure that our implementation is side-channel resistant in practice. 


\subsection{Implementation and Benchmarks}

We implemented our masked version of Picnic signing and benchmarked it on the ARM Cortex M4, using the pqm4 [KRSS] suite and the STMicro developer board STM32F407GDISC1. This board has one MB of flash memory and 192KB of RAM and comes equipped with a true random number generator implemented as a hardware peripheral. The microcontroller clock frequency ranges from 24 to $168 \mathrm{MHz}$, so following standard practice our benchmarks were executed at the lowest frequency to avoid the impact of memory wait states [FA17, HL19].

Our implementation is derived from the Picnic optimized implementation, which is primarily optimized for x64 platforms, and is not well-optimized for the M4. As such, our implementation results aim to bound the overhead of masking countermeasures. We also focus only on first-order protection, i.e., the case $T=2$, and implement only the L1 parameter set picnic3-L1. We decided for first-order protection to show the general effectiveness of our countermeasure. Also, first-order protection already significantly raises the bar for attacks, as higher-order attacks require a much more sophisticated adversary and the attacks become very expensive due to the long traces. As most of our countermeasures are general, we expect them to apply equally to more optimized M4 implementations of Picnic, and (with some effort) to implementations of other MPCitH-based proof systems.

Since our masked implementation produces picnic3-L1 signatures compatible with the specified version [Pic20], we do not repeat signature or key sizes in our benchmarks: public keys are 34 bytes, secret keys are 17 bytes, signatures are $12.4 \mathrm{~KB}$. All other parameters such as number of MPC parties, MPC instances, digest lengths, etc. are as specified in [Pic20]. For reference, the verification time in our implementation is $204 \mathrm{M}$ cycles.

Masked Keccak. We implemented three different flavors of masked Keccak described in the last section: IND, DOM, and SNI. The implementation was built on top of the in-place 32-bit ARMv7-M assembly code found in the official Keccak code package ${ }^{3}$ (XKCP) to operate over a double-sized state storing the two shares. We implement the same Keccak API used in Picnic by replicating functions over each share of the state, and modifying the round function to implement the non-linear operations. Because of the larger state, additional pressure was put on the registers and several intermediate variables had to be spilled onto the stack. This caused some additional performance overhead beyond the raw cost of masking. In order to prevent leakage, we took additional care to rotate registers between rounds to prevent them from loading shares of the same variable [BDPA10].

Benchmarks. In Table 1 we give cycle counts for our masked implementation with various options for how the hash function calls are masked. The masking cost for the non-hashing operations is $158 \mathrm{M}$ cycles, which represents an overhead of $1.5 x$ over baseline. Since this is effectively doing the MPC simulation with 2-encoded values, we might expect a factor two slowdown rather than 1.5, however, this is explained by the fact that many of the operations to implement LowMC are ANDs and XORs with public constants, which are more efficient than operations on 2 -encoded values. Then the cost when masking the hashing naively (by masking all operations), is given for the three Keccak masking options (SNI, DOM and IND) and we see that the overhead is $1201 \mathrm{M}, 827 \mathrm{M}$ and $395 \mathrm{M}$ cycles respectively. By using our analysis of Section 5.2, and selectively masking only sensitive hash function calls, the overhead for IND drops to 153M cycles, and all the way down to $86 \mathrm{M}$ cycles when we additionally use the half-masking optimization. In this most performant case, we have roughly $2 / 3$ of the overhead accruing to the Picnic and MPC operations, and $1 / 3$ to the hashing.

Stack usage was essentially constant for all configurations we benchmarked, since the total amount of memory required is dominated by storing the signature, the commitment and seed trees and not by the storage space for intermediate values that we must $t$-encode.

\footnotetext{
${ }^{3}$ https://github.com/gvanas/KeccakCodePackage
} 
Table 1: Benchmarks in millions of Cortex-M4 cycles showing the masking overhead for types of side-channel protections. The options for Picnic are "No" masking and $T=2$ masking, as described in Section 5. For SHAKE, the "None" option indicates no masking is used for hash computations, "All-" prefix means every call is masked in one of three possible ways: SNI-secure $\left[\mathrm{BBD}^{+} 16\right]$, Domain-Oriented Masking (DOM) [GSM17] or using independent values (IND) [BDPA10]. "Selective" means that only sensitive calls are masked with independent values (as described in Section 5.2), and "Selective Half" means that in addition to Selectively masking and IND, we use half-masked SHAKE. The Hashing column provides the fraction of the signing time spent computing SHAKE.

\begin{tabular}{llrrrrrr}
\hline $\begin{array}{c}\text { Picnic } \\
\text { Masking }\end{array}$ & $\begin{array}{l}\text { SHAKE } \\
\text { Masking }\end{array}$ & $\begin{array}{c}\text { Signing } \\
\text { cycles }\end{array}$ & Hashing & $\begin{array}{c}\text { Masking } \\
\text { Overhead }\end{array}$ & Stack & Code & $\begin{array}{c}\text { Random } \\
\text { bytes (KB) }\end{array}$ \\
\hline No & None & 302 & $70 \%$ & 1.00 & 32,460 & 81,397 & 0 \\
\hline Yes & None & 460 & $50 \%$ & 1.52 & 32,516 & 103,114 & 2,025 \\
Yes & All-SNI & 1661 & $86 \%$ & 5.50 & 32,740 & 137,872 & 158,172 \\
Yes & All-DOM & 1287 & $81 \%$ & 4.26 & 32,740 & 130,432 & 80,378 \\
Yes & All-IND & 855 & $72 \%$ & 2.83 & 32,740 & 122,256 & 2,585 \\
Yes & Selective & 613 & $62 \%$ & 2.03 & 32,740 & 120,372 & 2,025 \\
Yes & Sel. Half & 546 & $57 \%$ & 1.81 & 32,740 & 120,372 & 2,025 \\
\hline
\end{tabular}

Code size increases by $1.5 \mathrm{x}$ in the most performant masked implementation (selective halfmasked), and by $1.7 \mathrm{x}$ in the fully SNI-secure version. Finally, the randomness requirements range from the baseline of $\approx 2 \mathrm{MB}$ when Keccak is masked with the IND method, to the much higher $80 \mathrm{MB}$ for the DOM method and $158 \mathrm{MB}$ for the SNI method, as these methods require additional refreshing of nonlinear operations within Keccak. Using the selective half-masking option reduces the randomness requirements of the DOM and SNI options significantly, since the number of hash function calls is decreased and some calls are only half-masked. By our estimate in Section 5.4 this would reduce randomness usage by about $65 \%$ for the DOM and SNI options. In terms of the $\approx 2 \mathrm{MB}$ of randomness used for the non-hashing operations, these are partly due to the refresh operations within the LowMC implementation (Line 11), they are required for NI security and since they did not have a significant impact on run time, we did not investigate the option of removing them. The other significant randomness consumer in the masked LowMC implementation is the masked AND operation (Algorithm 11). Here, future work could experiment with an implementation that masks ANDs as in the IND method for Keccak, with the aim of reducing randomness and improving run time.

\subsection{Experimental Leakage Analysis}

To ensure our masked implementations of Keccak and Picnic are practically side-channel resistant, we performed measurements of the implementation to confirm the absence of leakage. Our measurement setup comprises the STMicro developer board STM32F407GDISC1 also used for the performance benchmarks, operated at $168 \mathrm{MHz}$. We measure EM emanations using a Langer LF-U 2.5 near field probe connected to a Langer PA 303 preamplifier [EmP]. The EM probe is placed over the C29 blocking cap at a distance of approx. $1 \mathrm{~mm}$. Measurements are recorded using a Tektronix MSO 6. For the Keccak implementation, we sampled at $3.125 \mathrm{GS} / \mathrm{s}$ with a 12 bit resolution and $200 \mathrm{Mhz}$ bandwidth. For the Picnic measurements, which are 2 orders of magnitude longer, we reduced the sampling rate to $625 \mathrm{MS} / \mathrm{s}$ in order to obtain feasible measurement time and storage sizes. Note that this still over-samples the board $(168 \mathrm{MHz})$ by a rate of 3.7 which is well above the minimal oversampling threshold of 2 from he Nyquist-Shannon sampling theorem.

Masked Keccak Leakage Evaluation. For Keccak we evaluate the IND method and follow the FvR approach to detect all possible sources of first-order leakage. During the 

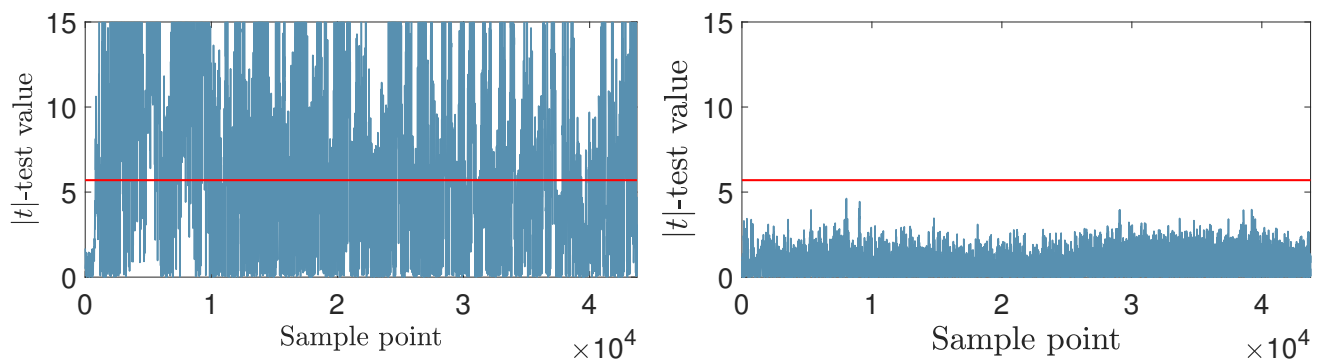

Figure 3: A first-order leakage detection test based on 2,000 traces on the protected Keccak implementation with fixed masking (left) and on 1,000,000 traces on the protected Keccak implementation (right). The threshold of $|t| \geq 5.7$ (as suggested by [DZD $\left.{ }^{+} 18\right]$ ) is violated throughout the implementation with fixed masking, indicating strong leakage. For the correctly masked implementation, the $|t|$-value remains below 5.7, even with 1,000,000 traces, indicating the absence of exploitable first-order leakage.
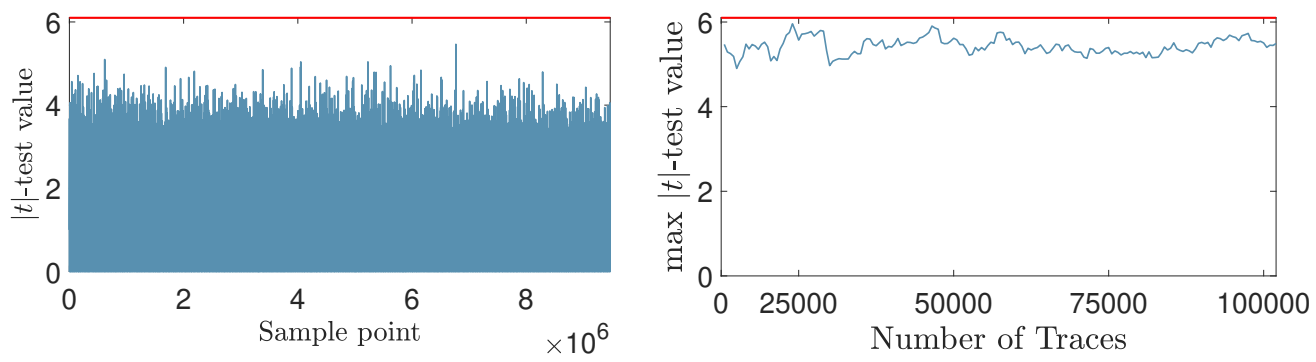

Figure 4: A first-order leakage detection test based on 100,000 traces on the Picnic3. The $|t|$-value remains below 6.1 (as suggested by [DZD $\left.{ }^{+} 18\right]$ ), indicating the absence of exploitable first-order leakage for the 100,000 traces. Also, the maximum $|t|$-value is bounded and becomes stable with the increased number of traces (right).

trace collection phase, a set of side-channel traces is collected by processing either a fixed input or a random input under the same conditions. The fixed or random choice for the input is made at random. After that, we calculate the means and standard deviations of the two side-channel trace sets separately. The $t$-test indicates whether the two distributions are indistinguishable for a first-order SCA. We apply the customary threshold values for long traces 5.7 as suggested by $\left[\mathrm{DZD}^{+} 18\right]$.

To show the sensitivity of the measurement setup for first-order leakage, we apply the test once to the correctly masked implementation and once to the same implementation with fixed masks. When masks are not chosen at random, the test must detect the resulting first-order leakage. The left hand side of the Fig. 3 shows the evaluation results of the masked Keccak implementation with fixed masks based on 2,000 measurement traces. As expected, the leakage test indicates strong leakage, with $|t|$ clearly above 5.7 . When masks are chosen uniformly at random, the $t$-value remains below 5.7 as shown in the right hand side of the Fig. 3, even if the number of measurement traces is increased to 1,000,000. We thus conclude that the masked Keccak implementation is secure and provides the expected resistance to first-order attacks.

Masked Picnic Leakage Evaluation. In order to analyze the leakage for the whole first-order masked Picnic3 implementation, we follow a similar methodology as for Keccak and employ the FvR approach. We collect the traces starting at the beginning of signature 
generation until the end of the first MPC instance, i.e., including a single preprocessing phase and simulation of an online phase(Line 1 to Line 22 in Algorithm 4). Note that after this point, everything is public, and any leakage gives no additional information beyond what is made public in the signature. To analyze our signature implementation, we choose the FvR key scenario, under randomized messages, as proposed in [TG16] for asymmetric cryptosystems. ${ }^{4}$ In addition we needed to add artificial wait cycles before accessing the board's hardware TRNG to ensure that we will never need varying amounts of time for it to become ready, as this would destroy the constant time property required for the TVLA. Note that this change is only necessary for the test setup and not required in the production code.

The sets of side-channel traces are collected by signing a random message using either a fixed key or a random key. As shown in Fig. 4, the $t$-value remains below 6.1 using 100,000 traces which indicates the absence of leakage. Moreover, the maximum $|t|$-value is indeed bounded and has a stable pattern. By contrast, an exploitable leakage as shown in Fig. 1 or Fig. 5 exceeds the threshold value within as few as 2,725 traces and has a clear increasing pattern. Thus we can conclude that the first-order masked Picnic3 implementation provides the expected SCA resistance.

Scaling to higher security levels and masking orders. Our implementation and experimental evaluation is limited to security level L1 and first-order protection (the case $T=2$ ). Since we expect the proportion of time spent on hashing vs. MPC simulation to be similar at levels L1 and L5 (as was the case for x64, see [KZ20, Table 7]), we expect the overhead of our masking techniques to be similar at L5 as well. When $T$ increases, we can only make rough predictions. We expect running time overhead to increase quadratically and memory overhead to increase linearly, due to the asymptotic behavior of masking nonlinear operations, and the additional storage required for $T$-encoded values.

\section{Conclusion and Future Work}

In this paper we study the side-channel security of MPCitH proof protocols and related signature schemes. We found and demonstrated a new probing attack on the KKW proof protocol (as implemented by Picnic). We then show that masking the signing operations is a practical countermeasure for side-channel attacks, and prove our masked KKW and Picnic3 meet the standard security notion (NIo), with a mix of both manual proofs and formal verification with the maskVerif tool.

We implemented multiple masked versions of the Picnic3 signature scheme for the ARM Cortex M4 as a case study, and found that the cost of masking (in terms of runtime) is high when we simply apply SNI-secure masking to all hashing operations. After careful analysis of the hashing operations, we found that the masking overhead can be made quite reasonable (as low as 1.8x) under modest assumptions that we verified with practical leakage analysis of our implementation. We recommend this version with selective half-masking as the best security-efficiency trade-off among our implementations, and have configured it as the default in our software package. With hardware support for side-channel protected hashing, our work shows that the overhead of masking the non-hashing parts of Picnic signing is about $1.5 \mathrm{x}$, and our NI analysis applies here.

Our flexible masked SHA-3 implementation is the first publicly available one, and will be useful to other projects as SHA-3 becomes more common. We also expect our

\footnotetext{
${ }^{4}$ The FvR-message scenario with a fixed key is not a good fit for randomized signature schemes like Picnic3 where the entire internal state is randomized even for fixed messages. If the randomness is fixed (by fixing $R$ in Line 1 of Algorithm 4), the signature scheme becomes artificially deterministic. Then several public parts of the signature generation process, including the signature itself, will be picked up by the TVLA test. Similarly, in the deterministic case the root seed is fixed for fixed messages (and random for random ones), also creating leakage which does not exist for randomized signatures. We still performed the analysis and verified that leakage only occurs in such expected places.
} 
half-masking optimization to find application in other implementations, as most hash operations have a non-sensitive input or output.

Performance improvements (while maintaining resistance to side-channel attacks) are an obvious direction for future work, both on the M4 and other embedded platforms. Reducing the amount of randomness consumed by our mitigations is also an interesting way to improve performance, together with generalizing to higher order protection efficiently.

Finally, an implementation that combines SCA resistance and resistance to fault attacks (perhaps leveraging the fault-resistance results for Picnic in [AOTZ20]) would also make interesting follow-up work.

\section{Acknowledgments}

We thank Daniel Kales and Sebastian Ramacher for help porting the optimized Picnic implementation to the M4. We thank the anonymous reviewers of TCHES for their detailed comments. This work has been supported by: the German Federal Ministry of Education and Research (BMBF) under the projects PQC4MED (FKZ 16KIS1045) and Jupiter (FKZ 16ME0234); the Concordium Blockhain Research Center, Aarhus University, Denmark; the Carlsberg Foundation under the Semper Ardens Research Project CF18-112 (BCM); the European Research Council (ERC) under the European Unions's Horizon 2020 research and innovation programme under grant agreement No 803096 (SPEC).

\section{References}

[AOTZ20] Diego F. Aranha, Claudio Orlandi, Akira Takahashi, and Greg Zaverucha. Security of hedged Fiat-Shamir signatures under fault attacks. In Anne Canteaut and Yuval Ishai, editors, EUROCRYPT 2020, Part I, volume 12105 of LNCS, pages 644-674. Springer, Heidelberg, May 2020.

[ARS ${ }^{+}$15] Martin R. Albrecht, Christian Rechberger, Thomas Schneider, Tyge Tiessen, and Michael Zohner. Ciphers for MPC and FHE. In Elisabeth Oswald and Marc Fischlin, editors, EUROCRYPT 2015, Part I, volume 9056 of LNCS, pages 430-454. Springer, Heidelberg, April 2015.

$\left[\mathrm{BBC}^{+} 19\right]$ Gilles Barthe, Sonia Belaïd, Gaëtan Cassiers, Pierre-Alain Fouque, Benjamin Grégoire, and François-Xavier Standaert. maskVerif: Automated verification of higher-order masking in presence of physical defaults. In Kazue Sako, Steve Schneider, and Peter Y. A. Ryan, editors, ESORICS 2019, Part I, volume 11735 of LNCS, pages 300-318. Springer, Heidelberg, September 2019.

$\left[\mathrm{BBD}^{+} 15 \mathrm{a}\right]$ Gilles Barthe, Sonia Belaïd, François Dupressoir, Pierre-Alain Fouque, Benjamin Grégoire, and Pierre-Yves Strub. Verified proofs of higher-order masking. In Elisabeth Oswald and Marc Fischlin, editors, EUROCRYPT 2015, Part I, volume 9056 of LNCS, pages 457-485. Springer, Heidelberg, April 2015.

$\left[\mathrm{BBD}^{+} 15 \mathrm{~b}\right]$ Gilles Barthe, Sonia Belaïd, François Dupressoir, Pierre-Alain Fouque, Benjamin Grégoire, Pierre-Yves Strub, and Rébecca Zucchini. Compositional verification of higher-order masking: Application to a verifying masking compiler. Cryptology ePrint Archive, Report 2015/506, 2015. https://eprint.iacr.org/eprint-bin/getfile.pl?entry=2015/ 506\&version $=20150527: 192221 \& f i l e=506 . p d f$.

$\left[\mathrm{BBD}^{+} 16\right]$ Gilles Barthe, Sonia Belaïd, François Dupressoir, Pierre-Alain Fouque, Benjamin Grégoire, Pierre-Yves Strub, and Rébecca Zucchini. Strong noninterference and type-directed higher-order masking. In Edgar R. Weippl, 
D. Aranha, S. Berndt, T. Eisenbarth, O. Seker, A. Takahashi, L. Wilke, G. Zaveruch 261

Stefan Katzenbeisser, Christopher Kruegel, Andrew C. Myers, and Shai Halevi, editors, ACM CCS 2016, pages 116-129. ACM Press, October 2016.

$\left[\mathrm{BBE}^{+} 18\right] \quad$ Gilles Barthe, Sonia Belaïd, Thomas Espitau, Pierre-Alain Fouque, Benjamin Grégoire, Mélissa Rossi, and Mehdi Tibouchi. Masking the GLP lattice-based signature scheme at any order. In Jesper Buus Nielsen and Vincent Rijmen, editors, EUROCRYPT 2018, Part II, volume 10821 of LNCS, pages 354-384. Springer, Heidelberg, April / May 2018.

[BBE $\left.{ }^{+} 19\right] \quad$ Gilles Barthe, Sonia Belaïd, Thomas Espitau, Pierre-Alain Fouque, Mélissa Rossi, and Mehdi Tibouchi. GALACTICS: Gaussian sampling for latticebased constant- time implementation of cryptographic signatures, revisited. In Lorenzo Cavallaro, Johannes Kinder, XiaoFeng Wang, and Jonathan Katz, editors, ACM CCS 2019, pages 2147-2164. ACM Press, November 2019.

[BDL97] Dan Boneh, Richard A. DeMillo, and Richard J. Lipton. On the importance of checking cryptographic protocols for faults (extended abstract). In Walter Fumy, editor, EUROCRYPT'97, volume 1233 of LNCS, pages 37-51. Springer, Heidelberg, May 1997.

[BDPA10] Guido Bertoni, Joan Daemen, Michael Peeters, and Gilles Van Assche. Building power analysis resistant implementations of keccak. Second SHA-3 Candidate Conference, 2010. https://keccak.team/files/KeccakDPA.pdf.

[Bea92] Donald Beaver. Efficient multiparty protocols using circuit randomization. In Joan Feigenbaum, editor, CRYPTO'91, volume 576 of LNCS, pages 420-432. Springer, Heidelberg, August 1992.

[Beu20] Ward Beullens. Sigma protocols for MQ, PKP and SIS, and Fishy signature schemes. In Anne Canteaut and Yuval Ishai, editors, EUROCRYPT 2020, Part III, volume 12107 of LNCS, pages 183-211. Springer, Heidelberg, May 2020.

[BN20] Carsten Baum and Ariel Nof. Concretely-efficient zero-knowledge arguments for arithmetic circuits and their application to lattice-based cryptography. In Aggelos Kiayias, Markulf Kohlweiss, Petros Wallden, and Vassilis Zikas, editors, PKC 2020, Part I, volume 12110 of LNCS, pages 495-526. Springer, Heidelberg, May 2020.

$\left[\mathrm{CDG}^{+} 17\right]$ Melissa Chase, David Derler, Steven Goldfeder, Claudio Orlandi, Sebastian Ramacher, Christian Rechberger, Daniel Slamanig, and Greg Zaverucha. Post-quantum zero-knowledge and signatures from symmetric-key primitives. In Bhavani M. Thuraisingham, David Evans, Tal Malkin, and Dongyan Xu, editors, ACM CCS 2017, pages 1825-1842. ACM Press, October / November 2017.

[CGPZ16] Jean-Sébastien Coron, Aurélien Greuet, Emmanuel Prouff, and Rina Zeitoun. Faster evaluation of SBoxes via common shares. In Benedikt Gierlichs and Axel Y. Poschmann, editors, CHES 2016, volume 9813 of LNCS, pages 498-514. Springer, Heidelberg, August 2016.

[CJRR99a] Suresh Chari, Charanjit S. Jutla, Josyula R. Rao, and Pankaj Rohatgi. Towards sound approaches to counteract power-analysis attacks. In Michael J. Wiener, editor, CRYPTO'99, volume 1666 of LNCS, pages 398-412. Springer, Heidelberg, August 1999. 
[CJRR99b] Suresh Chari, Charanjit S. Jutla, Josyula R. Rao, and Pankaj Rohatgi. Towards sound approaches to counteract power-analysis attacks. In CRYPTO, volume 1666 of Lecture Notes in Computer Science, pages 398-412. Springer, 1999.

[CS20] Gaëtan Cassiers and François-Xavier Standaert. Trivially and efficiently composing masked gadgets with probe isolating non-interference. IEEE Transactions on Information Forensics and Security, 15:2542-2555, 2020.

[Dae17] Joan Daemen. Changing of the guards: A simple and efficient method for achieving uniformity in threshold sharing. In Wieland Fischer and Naofumi Homma, editors, CHES 2017, volume 10529 of $L N C S$, pages 137-153. Springer, Heidelberg, September 2017.

[Dam10] Ivan Damgård. On $\Sigma$-protocols. http://www.cs.au.dk/ ivan/Sigma.pdf, 2010 .

[DDF19] Alexandre Duc, Stefan Dziembowski, and Sebastian Faust. Unifying leakage models: From probing attacks to noisy leakage. Journal of Cryptology, 32(1):151-177, January 2019.

[dDOS19] Cyprien de Saint Guilhem, Lauren De Meyer, Emmanuela Orsini, and Nigel P. Smart. BBQ: Using AES in picnic signatures. In Kenneth G. Paterson and Douglas Stebila, editors, SAC 2019, volume 11959 of $L N C S$, pages 669-692. Springer, Heidelberg, August 2019.

[DN19] Itai Dinur and Niv Nadler. Multi-target attacks on the Picnic signature scheme and related protocols. In Yuval Ishai and Vincent Rijmen, editors, EUROCRYPT 2019, Part III, volume 11478 of LNCS, pages 699-727. Springer, Heidelberg, May 2019.

[DOTT21] Ivan Damgård, Claudio Orlandi, Akira Takahashi, and Mehdi Tibouchi. Two-round n-out-of-n and multi-signatures and trapdoor commitment from lattices. In Juan Garay, editor, PKC 2021, Part I, volume 12710 of LNCS, pages 99-130. Springer, Heidelberg, May 2021.

[DPSZ12] Ivan Damgård, Valerio Pastro, Nigel P. Smart, and Sarah Zakarias. Multiparty computation from somewhat homomorphic encryption. In Reihaneh SafaviNaini and Ran Canetti, editors, CRYPTO 2012, volume 7417 of LNCS, pages 643-662. Springer, Heidelberg, August 2012.

[DZ13] Ivan Damgård and Sarah Zakarias. Constant-overhead secure computation of Boolean circuits using preprocessing. In Amit Sahai, editor, TCC 2013, volume 7785 of LNCS, pages 621-641. Springer, Heidelberg, March 2013.

$\left[\mathrm{DZD}^{+} 18\right]$ A. Adam Ding, Liwei Zhang, Francois Durvaux, Francois-Xavier Standaert, and Yunsi Fei. Towards sound and optimal leakage detection procedure. In Thomas Eisenbarth and Yannick Teglia, editors, Smart Card Research and Advanced Applications, pages 105-122, Cham, 2018. Springer International Publishing.

[EmP $]$ LF-U 2.5, H-Field Probe $100 \mathrm{kHz}-50 \mathrm{MHz}$. https://www . langer-emv.de/en/product/lf-passive-100-khz-50-mhz/36/

lf-u-2-5-h-field-probe-100-khz-up-to-50-mhz/5. 
[EvMY14] Thomas Eisenbarth, Ingo von Maurich, and Xin Ye. Faster hash-based signatures with bounded leakage. In Tanja Lange, Kristin Lauter, and Petr Lisonek, editors, SAC 2013, volume 8282 of LNCS, pages 223-243. Springer, Heidelberg, August 2014.

[FA17] Hayato Fujii and Diego F. Aranha. Curve25519 for the cortex-M4 and beyond. In Tanja Lange and Orr Dunkelman, editors, LATINCRYPT 2017, volume 11368 of LNCS, pages 109-127. Springer, Heidelberg, September 2017.

[FS87] Amos Fiat and Adi Shamir. How to prove yourself: Practical solutions to identification and signature problems. In Andrew M. Odlyzko, editor, CRYPTO'86, volume 263 of LNCS, pages 186-194. Springer, Heidelberg, August 1987.

[GJJR11] Gilbert Goodwill, Benjamin Jun, Josh Jaffe, and Pankaj Rohatgi. A testing methodology for side-channel resistance validation, 2011. NIST non-invasive attack testing workshop, https://csrc.nist.gov/csrc/media/events/ non-invasive-attack-testing-workshop/documents/08_goodwill.pdf.

[GMO16] Irene Giacomelli, Jesper Madsen, and Claudio Orlandi. ZKBoo: Faster zeroknowledge for Boolean circuits. In Thorsten Holz and Stefan Savage, editors, USENIX Security 2016, pages 1069-1083. USENIX Association, August 2016.

[GR19] François Gérard and Mélissa Rossi. An efficient and provable masked implementation of qtesla. In Sonia Belaïd and Tim Güneysu, editors, Smart Card Research and Advanced Applications - 18th International Conference, CARDIS 2019, Prague, Czech Republic, November 11-13, 2019, Revised Selected Papers, volume 11833 of Lecture Notes in Computer Science, pages 74-91. Springer, 2019.

[GSDM $\left.{ }^{+} 19\right]$ Hannes Gross, Ko Stoffelen, Lauren De Meyer, Martin Krenn, and Stefan Mangard. First-order masking with only two random bits. In Proceedings of ACM Workshop on Theory of Implementation Security Workshop, TIS'19, page 10-23, New York, NY, USA, 2019. Association for Computing Machinery.

[GSE20] Tim Gellersen, Okan Seker, and Thomas Eisenbarth. Differential power analysis of the Picnic signature scheme. To appear at PQCrypto 2021. Cryptology ePrint Archive, Report 2020/267, 2020. https://eprint.iacr. org/2020/267.

[GSM17] Hannes Groß, David Schaffenrath, and Stefan Mangard. Higher-order sidechannel protected implementations of KECCAK. In DSD, pages 205-212. IEEE Computer Society, 2017.

[HL19] Björn Haase and Benoît Labrique. AuCPace: Efficient verifier-based PAKE protocol tailored for the IIoT. IACR TCHES, 2019(2):1-48, 2019. https: //tches.iacr.org/index.php/TCHES/article/view/7384.

[IKOS07] Yuval Ishai, Eyal Kushilevitz, Rafail Ostrovsky, and Amit Sahai. Zeroknowledge from secure multiparty computation. In David S. Johnson and Uriel Feige, editors, 39th ACM STOC, pages 21-30. ACM Press, June 2007.

[ISW03] Yuval Ishai, Amit Sahai, and David Wagner. Private circuits: Securing hardware against probing attacks. In Dan Boneh, editor, CRYPTO 2003, volume 2729 of $L N C S$, pages 463-481. Springer, Heidelberg, August 2003. 
$\left[\mathrm{KGB}^{+} 18\right]$ Matthias J. Kannwischer, Aymeric Genêt, Denis Butin, Juliane Krämer, and Johannes Buchmann. Differential power analysis of XMSS and SPHINCS. In Junfeng Fan and Benedikt Gierlichs, editors, COSADE 2018, volume 10815 of LNCS, pages 168-188. Springer, Heidelberg, April 2018.

$\left[\mathrm{KGM}^{+} 20\right]$ Thilo Krachenfels, Fatemeh Ganji, Amir Moradi, Shahin Tajik, and JeanPierre Seifert. Real-world snapshots vs. theory: Questioning the t-probing security model, 2020. To appear at IEEE S\&P 2021.

[KJJ99] Paul C. Kocher, Joshua Jaffe, and Benjamin Jun. Differential power analysis. In Michael J. Wiener, editor, CRYPTO'99, volume 1666 of LNCS, pages 388-397. Springer, Heidelberg, August 1999.

[KKW18] Jonathan Katz, Vladimir Kolesnikov, and Xiao Wang. Improved noninteractive zero knowledge with applications to post-quantum signatures. In David Lie, Mohammad Mannan, Michael Backes, and XiaoFeng Wang, editors, ACM CCS 2018, pages 525-537. ACM Press, October 2018.

[Koc96] Paul C. Kocher. Timing attacks on implementations of Diffie-Hellman, RSA, DSS, and other systems. In Neal Koblitz, editor, CRYPTO'96, volume 1109 of LNCS, pages 104-113. Springer, Heidelberg, August 1996.

[KPP20] Matthias J. Kannwischer, Peter Pessl, and Robert Primas. Single-trace attacks on Keccak. IACR TCHES, 2020(3):243-268, 2020. https://tches . iacr.org/index.php/TCHES/article/view/8590.

[KRSS] Matthias J. Kannwischer, Joost Rijneveld, Peter Schwabe, and Ko Stoffelen. PQM4: Post-quantum crypto library for the ARM Cortex-M4. https: //github.com/mupq/pqm4.

[KZ20] Daniel Kales and Greg Zaverucha. Improving the performance of the Picnic signature scheme. IACR TCHES, 2020(4):154-188, 2020. https://tches . iacr.org/index.php/TCHES/article/view/8680.

[Lyu09] Vadim Lyubashevsky. Fiat-Shamir with aborts: Applications to lattice and factoring-based signatures. In Mitsuru Matsui, editor, ASIACRYPT 2009, volume 5912 of $L N C S$, pages 598-616. Springer, Heidelberg, December 2009.

[MGTF19] Vincent Migliore, Benoît Gérard, Mehdi Tibouchi, and Pierre-Alain Fouque. Masking Dilithium - efficient implementation and side-channel evaluation. In Robert H. Deng, Valérie Gauthier-Umaña, Martín Ochoa, and Moti Yung, editors, ACNS 19, volume 11464 of LNCS, pages 344-362. Springer, Heidelberg, June 2019.

[Nat20] National Institute of Standards and Technology. Status Report on the Second Round of the NIST Post-Quantum Cryptography Standardization Process, 2020. Available at https://doi.org/10.6028/NIST.IR.8309.

[NNOB12] Jesper Buus Nielsen, Peter Sebastian Nordholt, Claudio Orlandi, and Sai Sheshank Burra. A new approach to practical active-secure two-party computation. In Reihaneh Safavi-Naini and Ran Canetti, editors, CRYPTO 2012, volume 7417 of $L N C S$, pages 681-700. Springer, Heidelberg, August 2012.

[Pic20] The Picnic Design Team. The Picnic Signature Algorithm Specification, April 2020. Version 3.0, Available at https://microsoft.github.io/Picnic/. 
[PR13] Emmanuel Prouff and Matthieu Rivain. Masking against side-channel attacks: A formal security proof. In Thomas Johansson and Phong Q. Nguyen, editors, EUROCRYPT 2013, volume 7881 of LNCS, pages 142-159. Springer, Heidelberg, May 2013.

[QS11] Jean-Jacques Quisquater and David Samyde. Electromagnetic attack. In Henk C. A. van Tilborg and Sushil Jajodia, editors, Encyclopedia of Cryptography and Security, 2nd Ed, pages 382-385. Springer, 2011.

[SBWE20] Okan Seker, Sebastian Berndt, Luca Wilke, and Thomas Eisenbarth. SNI-inthe-head: Protecting MPC-in-the-head protocols against side-channel analysis. In Jay Ligatti, Xinming Ou, Jonathan Katz, and Giovanni Vigna, editors, ACM CCS 20, pages 1033-1049. ACM Press, November 2020.

[SEL21] Okan Seker, Thomas Eisenbarth, and Maciej Liskiewicz. A white-box masking scheme resisting computational and algebraic attacks. IACR Transactions on Cryptographic Hardware and Embedded Systems, 2021(2):61-105, Feb. 2021.

[Ste94] Jacques Stern. A new identification scheme based on syndrome decoding. In Douglas R. Stinson, editor, CRYPTO'93, volume 773 of LNCS, pages 13-21. Springer, Heidelberg, August 1994.

[TG16] Michael Tunstall and Gilbert Goodwill. Applying TVLA to public key cryptographic algorithms. Cryptology ePrint Archive, Report 2016/513, 2016. https://eprint.iacr.org/2016/513.

$\left[\mathrm{VWA}^{+} 21\right]$ Benoit Viguier, David Wong, Gilles Van Assche, Quynh Dang, and Joan Daemen. KangarooTwelve. IRTF Crypto Forum Internet-Draft, Feb 2021. https://tools.ietf.org/html/draft-irtf-cfrg-kangarootwelve-05.

$\left[\mathrm{ZCD}^{+} 20\right] \quad$ Greg Zaverucha, Melissa Chase, David Derler, Steven Goldfeder, Claudio Orlandi, Sebastian Ramacher, Christian Rechberger, Daniel Slamanig, Jonathan Katz, Xiao Wang, Vladmir Kolesnikov, and Daniel Kales. Picnic. Technical report, National Institute of Standards and Technology, 2020. available at https://csrc.nist.gov/projects/post-quantum-cryptography/ round-3-submissions. 

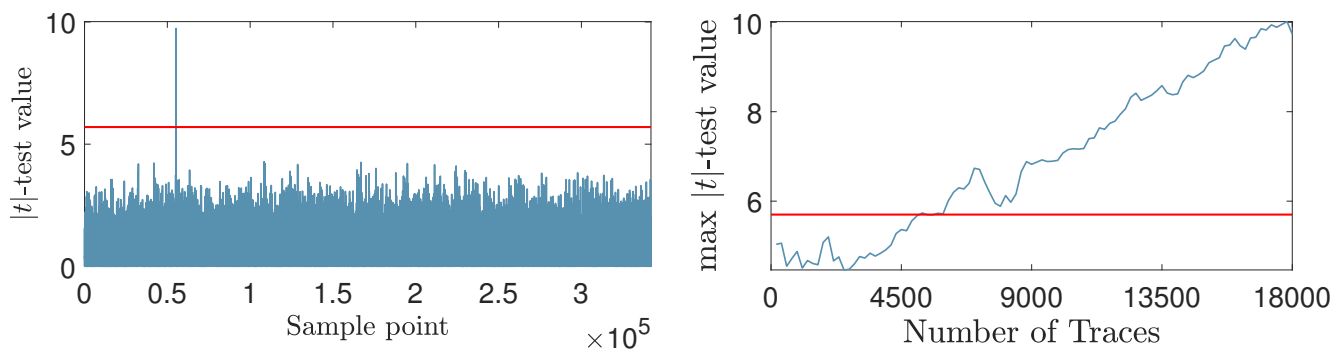

Figure 5: A first-order RvR test on the unprotected Picnic3 implementation using revealed values from the online phase. The traces are classified based on a single bit of $\hat{s k} K_{0}$. Values above the 5.7 threshold (red line) indicate there are strong leakages (left). Also, the maximum $|t|$-value increases with respect to number of traces and the leakage becomes absolute after 6,000 traces (right).

\section{A Probing the Unopened Party}

The second attack uses the revealed values for the online phase i.e. $\hat{w}^{(k)}$ and $\lambda_{i}^{w}$ for $i \neq i_{k}$. This attack is a straightforward variant of the one by Gellersen et al. [GSE20] and Seker et al. [SBWE20], but adapted to work with Picnic3. In contrast to the attack described above, we now target an MPC execution whose online phase is selected to be part of the signature (i.e., if $k \in \mathcal{C}$ ). In that case there is a single party $P_{i_{k}}$ whose internal state must remain hidden for the privacy of the MPC protocol to hold. By design, the values $\hat{w}^{(k)}$ and $\lambda_{i}^{w}$ for $i \neq i_{k}$ are revealed during the verification. Thus the measurements have a weak and noisy dependence to the value $\lambda_{i_{k}}^{w}$ which can be exploitable due to the revealed values.

We validate the attack using the same experimental setup and parameters as in Section 3.1. During the collection phase, we again process the Picnic3 with random messages with a fix secret key and measure the execution of the preprocessing phase (Algorithm 15), where the following is computed:

$$
\text { roundkey } \left._{0}=\lambda_{i_{k}}+\sum_{i \neq i_{k}} \lambda_{i} \text { and } \lambda^{s k}=\text { matMul( } \text { roundkey }_{0}, K_{0}^{-1}\right) \text {. }
$$

Since $\hat{s k}=s k+\lambda^{s k}$, we have the following equation for $\lambda_{i_{k}}$,

$$
\hat{s k}=s k+\left(\lambda_{i_{k}}+\sum_{i \neq i_{k}} \lambda_{i}\right) K_{0}^{-1}, \text { and } \lambda_{i_{k}}=(\hat{s k}-s k) K_{0}-\sum_{i \neq i_{k}} \lambda_{i} .
$$

Finally, we substitute the secret value $\lambda_{i_{k}}$ into Eq. (1),

$$
\text { roundkey }_{0}=(\hat{s k}-s k) K_{0}-\sum_{i \neq i_{k}} \lambda_{i}+\sum_{i \neq i_{k}} \lambda_{i}=(s k+\hat{s k}) K_{0}
$$

From Eq. (3) we observe that roundkey ${ }_{0}$ can be probed (over multiple traces) since $s k$ is a constant value. Then $\lambda_{i_{k}}$ can be calculated as roundkey ${ }_{0}-\sum_{i \neq i_{k}} \lambda_{i}$, and used to obtain the secret (as described above). The result of the analysis in Fig. 5 shows a clear dependence between the unrevealed value roundkey ${ }_{0}$ and the observable trace, as the $|t|$-value clearly exceeds 5.7 which shows an exploitable leakage.

\section{B Complete Description of the KKW Proof System}

In Fig. 6 we present a three-round KKW proof system. We remark that commitments (i.e., generation of $\operatorname{com}_{i}^{(k)}$ and com_on $\left.{ }^{(k)}\right)$ are de-randomized and replaced by a hash function as 
suggested in [KKW18, §3] (which loses HVZK but is still sufficient for provable security of signature), and the protocol is mildly generalized to work with arithmetic circuits according to [dDOS19, BN20].

Protocol. Below we present a basic framework for three-round MPCitH-PP proof systems. Here we describe the protocol for one MPC instance (with non-negligible soundness error) and in Fig. 6 we include a complete description of the KKW proof system that uses many instances in parallel (to achieve negligible soundness error). As the offline protocol proceeds independently of the secret witness, an MPCitH-PP prover can safely open the states of all $N$ parties for the verification of the preprocessing phase (i.e., triple generation).

(Commit) The prover $\mathrm{P}$ first samples a random seed for each $P_{i}$ and executes $\Pi_{C}^{\text {off }}$ to obtain the states of all $N$ parties after the offline phase. Then using these states and the masked witness $(\hat{w})_{w \in \mathbb{N}}$ as input, $\mathrm{P}$ executes $\Pi_{C}^{\circ}$ to obtain all broadcast messages observed during the online phase. Finally, $\mathrm{P}$ sends commitments to the states and broadcast messages to the verifier $\mathrm{V}$.

(Challenge) $\vee$ asks $\mathrm{P}$ to open either the offline or the online phase. For the latter case, $\mathrm{V}$ also randomly picks a party index $i^{\star}$, whose view is to remain hidden.

(Response) To open the offline phase, $\mathrm{P}$ sends all random seeds used during $\Pi_{C}^{\text {off }}$. To open the online phase, $\mathrm{P}$ sends broadcast messages coming from the party $P_{i^{\star}}$ during $\Pi_{C}^{\text {on }}$, as well as all the state information of the remaining $N-1$ parties.

(Verification) To check the offline phase, $\vee$ simply uses random seeds to execute $\Pi_{C}^{\text {off }}$ as $\mathrm{P}$ would, to obtain the resulting states of all $N$ parties. Then $\mathrm{V}$ checks that these states form a correct opening to the commitment of the offline phase. To check the online phase, $\vee$ simulates $\Pi_{C}^{\text {on }}$ with the broadcast messages from $P_{i^{\star}}$ and the states of the remaining $N-1$ parties as input, so as to obtain the broadcast messages of the other $N-1$ parties. Then, $\mathrm{V}$ checks that these broadcast messages form a correct opening to the commitments of the online phase.

\section{Our Protected Picnic3 Implementation}

In this section we give a detailed description of our masked Picnic3 implementation. Algorithm 4 has the top-level signature generation function, that calls the other algorithms in this section. Fig. 7 gives an overview of the optimized hashing operations mentioned in Section 5.2, indicating which optimizations are applied to each one.

Notation. $T$ is the number of shares used by our implementation, and masked values will be $T$-encoded. For LowMC, $n$ is the blocksize, and the precomputed constants $K_{i}, L_{i}$ and $R_{i}$ are as defined in Appendix F. The parameter $N$ is the number of MPC parties, and $M$ is the number of MPC instances.

\section{Data Types and Helper functions.}

- $T$-encoding: an additive secret sharing in $G F(2)$. For a bit $b$, the $T$-encoding is a vector of $T$ values $b_{1}, \ldots, b_{T}$ such that $b=\sum_{i=1}^{T} b_{i}$. For a bitstring $s$, the $T$-encoding is $T$ bitstrings over $G F(2)^{|s|}$ that XOR to $s$. As in other parts of the paper we use $\langle b\rangle$ to indicate that $b$ is $T$-encoded.

- $\oplus_{T}$ : XOR operation of $T$-encoded values. For two $T$-encoded inputs $\langle a\rangle$ and $\langle b\rangle$, we define $\langle c\rangle=\langle a\rangle \oplus_{T}\langle b\rangle$ as $c_{i}=a_{i} \oplus b_{i}$ for $i=1, \ldots, T$; for one $T$-encoded input XOR'd by a non-encoded constant $b$, we define $\langle c\rangle=\langle a\rangle \oplus_{T} b$ as $c_{1}=a_{1} \oplus b$ and $c_{i}=a_{i}$ for $i=2, \ldots, T$.

- SMul: AND operation of two T-encoded values. We use Algorithm 11. For example, with 2-encoded inputs $\langle a\rangle$ and $\langle b\rangle$, this algorithm outputs $\langle c\rangle=$ 


\section{Protocol KKW}

Inputs Both prover and verifier receive circuit $C$ as a statement. The prover also holds a witness $\mathbf{w}=(w)_{w \in \mathbb{N}}$ such that $C(\mathbf{w})=1$. Values $M, N, \tau$ are parameters of the protocol.

Commit 1. For each $k \in[M]$, the prover:

(a) Choose a uniform seed ${ }^{(k)}$ and use to generate values $\left\{\operatorname{seed}_{i}^{(k)}\right\}_{i \in[N]}$. Also the prover computes aux ${ }^{(k)} \in \mathbb{F}^{|C|}$ by running the offline phase of MPC $\Pi_{C}^{\text {off }}$ on input $\left\{\operatorname{seed}_{i}^{(k)}\right\}_{i \in[N]}$. For all $i=1, \ldots N-1$, let state ${ }_{i}^{(k)}=\operatorname{seed}_{i}^{(k)}$ and let $\operatorname{state}_{N}^{(k)}=\operatorname{seed}_{N}^{(k)} \| \operatorname{aux}^{(k)}$.

(b) Commit to the offline phase:

$$
\begin{aligned}
\operatorname{com}_{i}^{(k)} & =\mathrm{H}\left(\operatorname{state}_{i}^{(k)}\right) \text { for all } i \in[N] \\
\text { com_off }^{(k)} & =\mathrm{H}\left(\operatorname{com}_{1}^{(k)}, \ldots, \operatorname{com}_{N}^{(k)}\right) .
\end{aligned}
$$

(c) Compute the masked witness $\hat{w}^{(k)}=\lambda_{1}^{w}+\ldots+\lambda_{N}^{w}+w$ for each $w \in \mathbf{I N}$, where each $\lambda_{i}^{w}$ corresponds to party $P_{i}$ 's random share to mask the witness, and is read out from state $_{i}^{(k)}$

(d) Simulate the online phase of the $N$-party protocol by running the offline phase of MPC $\Pi_{C}^{\text {on }}$ on input $\left(\hat{w}^{(k)}\right)_{w \in \mathbb{N}}$ and $\left\{\text { state }_{i}^{(k)}\right\}_{i \in[N]}$, to produce $\left\{\operatorname{msgs}_{i}^{(k)}\right\}_{i \in[N]}$.

(e) Commit to the online phase:

$$
\text { com_on }^{(k)}=\mathrm{H}\left(\left\{\hat{w}^{(k)}\right\}_{w \in \mathbb{N}}, \text { msgs }_{1}^{(k)}, \ldots, \text { msgs }_{N}^{(k)}\right) .
$$

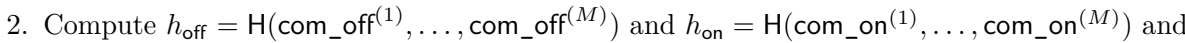
send $h^{*}=\mathrm{H}\left(h_{\text {off }}, h_{\text {on }}\right)$ to the verifier.

Challenge The prover receives the following challenges from the verifier: a uniform $\tau$-sized set $\mathcal{C} \subset[M]$ and $\mathcal{P}=\left\{i_{k}\right\}_{k \in \mathcal{C}}$ where each $i_{k} \in[N]$ is uniform.

Response For each $k \in[M] \backslash \mathcal{C}$, the prover sends seed ${ }^{(k)}$ and com_on ${ }^{(k)}$ for all to the verifier. For each $k \in \mathcal{C}$, the prover sends $\operatorname{com}_{i_{k}}^{(k)},\left\{\text { state }_{i}^{(k)}\right\}_{i \neq i_{k}},\left\{\hat{w}^{(k)}\right\}_{w \in \mathbb{N}}$ and $\operatorname{msgs}_{i_{k}}^{(k)}$ to the verifier.

Verification The verifier accepts if and only if all the following checks succeed:

1. Check the offline phase:

(a) For every $k \in \mathcal{C}$ and $i \neq i_{k}$, the verifier uses state ${ }_{i}^{(k)}$ to compute $\operatorname{com}_{i}^{(k)}$ as the prover would. Then produce com_off ${ }^{(k)}=\mathrm{H}\left(\operatorname{com}_{1}^{(k)}, \ldots, \operatorname{com}_{N}^{(k)}\right)$ using the received value $\operatorname{com}_{i_{k}}^{(k)}$,

(b) For every $k \in[M] \backslash \mathcal{C}$ the verifier uses seed ${ }^{(k)}$ to compute com_off ${ }^{(k)}$ as the prover would.

(c) Finally, the verifier computes $h_{\text {off }}=\mathrm{H}\left(\right.$ com_off $^{(1)}, \ldots$, com_off $\left.^{(M)}\right)$

2. Check the online phase:

(a) For $k \in \mathcal{C}$ the verifier simulates the online phase using $\left\{\operatorname{state}_{i}^{(k)}\right\}_{i \neq i_{k}}$, masked witness $\left(\hat{w}^{(k)}\right)_{w \in \mathbb{N}}$ and $\operatorname{msgs}_{i_{k}}^{(k)}$ to compute $\left\{\operatorname{msgs}_{i}\right\}_{i \neq i_{k}}$. Then compute com_on ${ }^{(k)}$ as if the prover would do.

(b) The verifier computes $h_{\mathrm{on}}=\mathrm{H}\left(\right.$ com_on $^{(1)}, \ldots$, com_on $\left.^{(M)}\right)$ using the received com_on ${ }^{(k)}$ for $k \in[M] \backslash \mathcal{C}$.

3. The verifier checks that $\mathbf{H}\left(h_{\text {off }}, h_{\text {on }}\right) \stackrel{?}{=} h^{*}$.

Figure 6: 3-round $\mathrm{KKW}$ proof system for an arithmetic circuit $C$ defined over $\mathbb{F}$. 


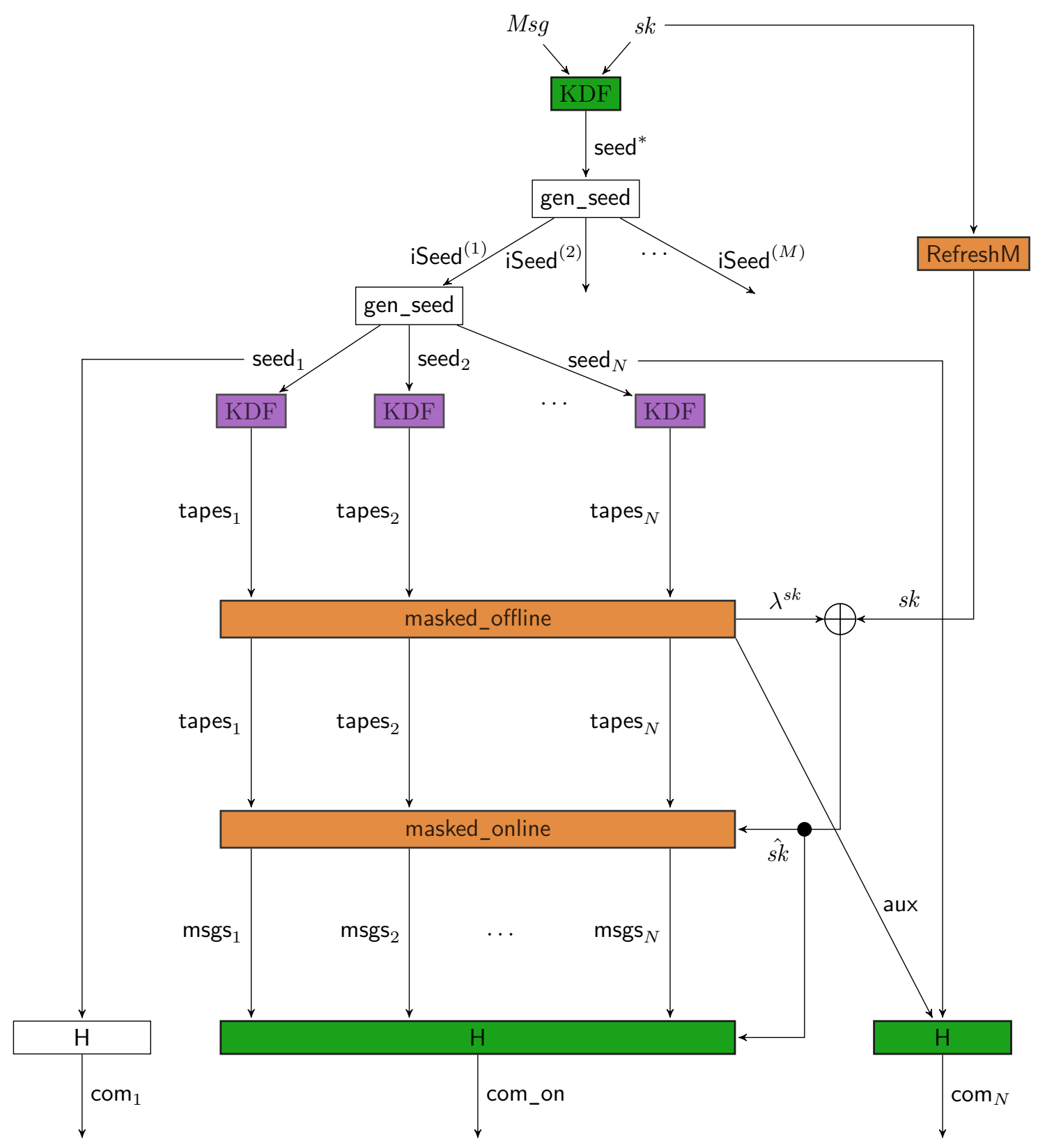

Figure 7: Summary of our masking protections and hashing optimizations from Section 5.2. The figure is fully expanded for one of the $M$ MPC instances, and shows the signer's operations for the commit phase of the protocol (i.e., before the challenge is computed). Hash functions in green are half-masked hash with sensitive inputs, hash functions in purple are half-masked with sensitive outputs, functions in orange are NI/SNI-secure gadgets, and the white functions are unprotected. The secret key (witness) is denoted $s k$ and $M s g$ is the message to be signed. In the figure, we omit hashing of $\left(\mathrm{com}_{1}, \ldots, \mathrm{com}_{N}\right)$ into com_off, since the inputs are public values that can be reconstructed from the signature, and therefore the hash computation is unmasked regardless of our optimization. 
$\operatorname{SMul}(\langle a\rangle,\langle b\rangle)$ as

$$
\begin{aligned}
& c_{1}=a_{1} b_{1}+r \\
& c_{2}=a_{2} b_{2}+a_{1} b_{2}+r+a_{2} b_{1}
\end{aligned}
$$

where $r$ is a fresh random bit. Note that $c=c_{1}+c_{2}=\left(a_{1}+a_{0}\right)\left(b_{1}+b_{0}\right)=a b$.

- Additional functions: Two additional helper functions from the literature are described in Appendix D. These are for refreshing the randomness of a $T$-encoded value and decoding (or unmasking) a $T$-encoded value.

- $\operatorname{matMul}_{T}$ : This is a generalization of the matMul matrix multiplication function in [Pic20, §6.4.4], modified to work on $T$-encoded input vectors. The matrix remains unshared. The input is a $T$-encoded vector $v$ of length $n$, a $n \times n$ matrix $M$, and the output is the length- $n$ vector $v M$. If $T=2$, we have $v=\left(v_{1}, v_{2}\right)$, the output is $\left(v_{1} M, v_{2} M\right)=\left(\operatorname{matMul}\left(v_{1}, M\right), \operatorname{matMul}\left(v_{2}, M\right)\right)$

- tapes: an object representing the $N$ random tapes, one per party. In case we need to be explicit about individual per-party tapes, it is parsed as tapes $=\operatorname{tape}_{1}\|\ldots,\| \operatorname{tape}_{N}$. Each tape is expanded from a seed masked version of SHAKE that produces $T$ encoded outputs, and we store the $T$-encoded outputs. We also store a $T$-encoded representation of the the aux tape, the $N$-th party's share.

- tapes_to_word(tapes, offset): Read one bit from each of the $N$ tapes at the index offset, and output an $N$-bit word. When the tapes are $T$-encoded, the output word is also $T$-encoded.

- tapes_to_parity ${ }_{T}(n$, tapes, offset): Reads $n$ bits from each tape at the index offset, the strings $s_{1}, \ldots, s_{N}$, returns a $T$-encoding of of $\bigoplus_{i=1}^{N} s_{i}$. Our implementation computes and stores a $T$-encoding of the parity tape (i.e., the XOR of all $N$ tapes) and uses this to implement the tapes_to_parity function.

\section{C.1 Specification of Fully Masked Picnic3}

Algorithm 4 specifies the masked Picnic3 signing operations without the half-masked hashing optimizations that we described in Section 5.2. The notation is as defined elsewhere in the paper, and in the appendix on LowMC (Appendix F). All functions marked in orange modify $T$-encoded values during the computation. The Unmask function takes a $T$-encoded value and returns the non-encoded value, by summing the shares after refresh (see Algorithm 13). We verified that Algorithm 4 is indeed NI secure with maskVerif for up to second order (implying it is also NIo for any public outputs).

\section{C.2 Simulation of the Offline Phase}

Algorithms 5 to 7 describe the protected preprocessing phase. The description is very similar to the preprocessing phase in the specification [Pic20, Section 7.4], however, the data types and helper functions are different. Primarily, all variables are $T$-encoded. Algorithm 6 describes our masked version of the LowMC S-box used for preprocessing (called by Algorithm 5), which in turn calls Algorithm 7 the AND operation for the preprocessing phase. Also, our presentation assumes that Algorithm 5 is used for signature generation only, since verification can use an unprotected implementation.

\section{C.3 Simulation of the Online Phase}

We now describe how the online phase of the MPC simulation is masked. Algorithm 8 is the MPC simulation for the online phase, implementing the LowMC circuit. For each AND gate, each party $i$ broadcasts a bit and these are output to msgs $_{i}$, these are also 
T-encoded. In Algorithm 9 we describe the S-box implementation used in Algorithm 8. Finally we have Algorithm 10 that describes the online simulation of an individual AND gate. The broadcast values (written to $\mathrm{msgs}_{i}$ ) and output bit are also $T$-encoded. Recall that SMul is implemented with the ISW multiplier (Algorithm 11).

Note that we need to refresh $T$-encoded st before each invocation of masked_sbox online (line 6 of Algorithm 8). On one hand, since every round of LowMC involves a linear transformation of st (line 1 and 7), every bit of st depends on all $n$ bits of the previous st, which corresponds to a problematic composition pattern mentioned in $\left[\mathrm{BBD}^{+} 15 \mathrm{~b}\right.$, Diagram 1]. On the other hand, all the other gadgets are in fact characterized as an affine gadget, which can be security composed in an arbitrary fashion. Hence, inserting RefreshM as we do is necessary and sufficient for the entire construction to be provably NIo secure.

Storage of secret keys. We assume that the Picnic secret key (a bitstring of length $n$ ), is stored in a $T$-encoded representation. Picnic key pair generation may be modified to generate $T$-encoded secret keys, or an implementation may use regular key generation in a trusted environment (e.g., during device manufacture), and then encode the secret key. As this is not important for performance, our implementation takes the regular key and $T$-encodes it at the beginning of signing. Then the input to MPC simulation is the $T$-encoded value $\langle\hat{s k}\rangle=\left\langle\lambda^{s k}\right\rangle \oplus_{T}\langle s k\rangle$, where $\left\langle\lambda^{s k}\right\rangle$ is the $T$-encoded random masks output by preprocessing. 


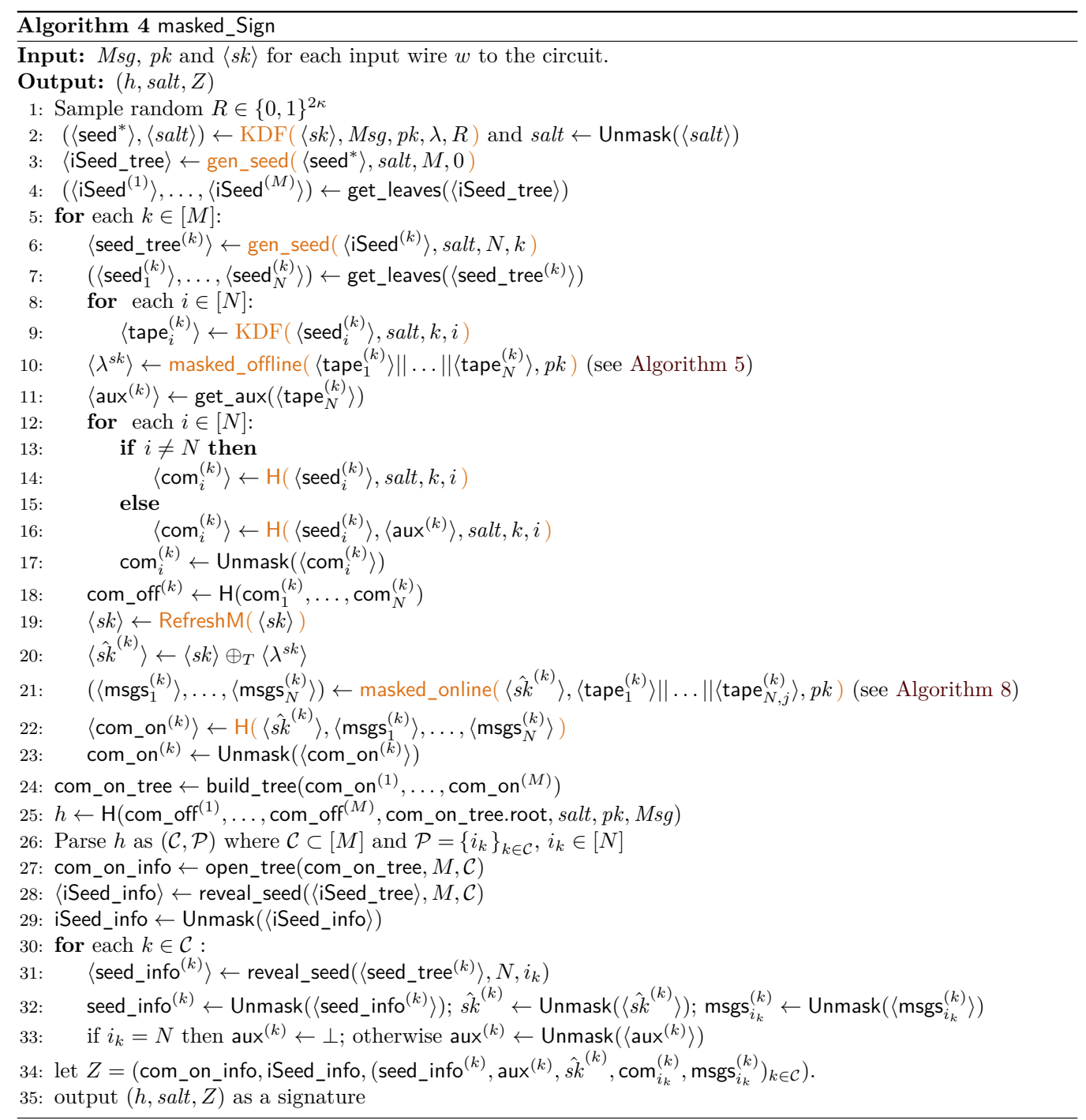



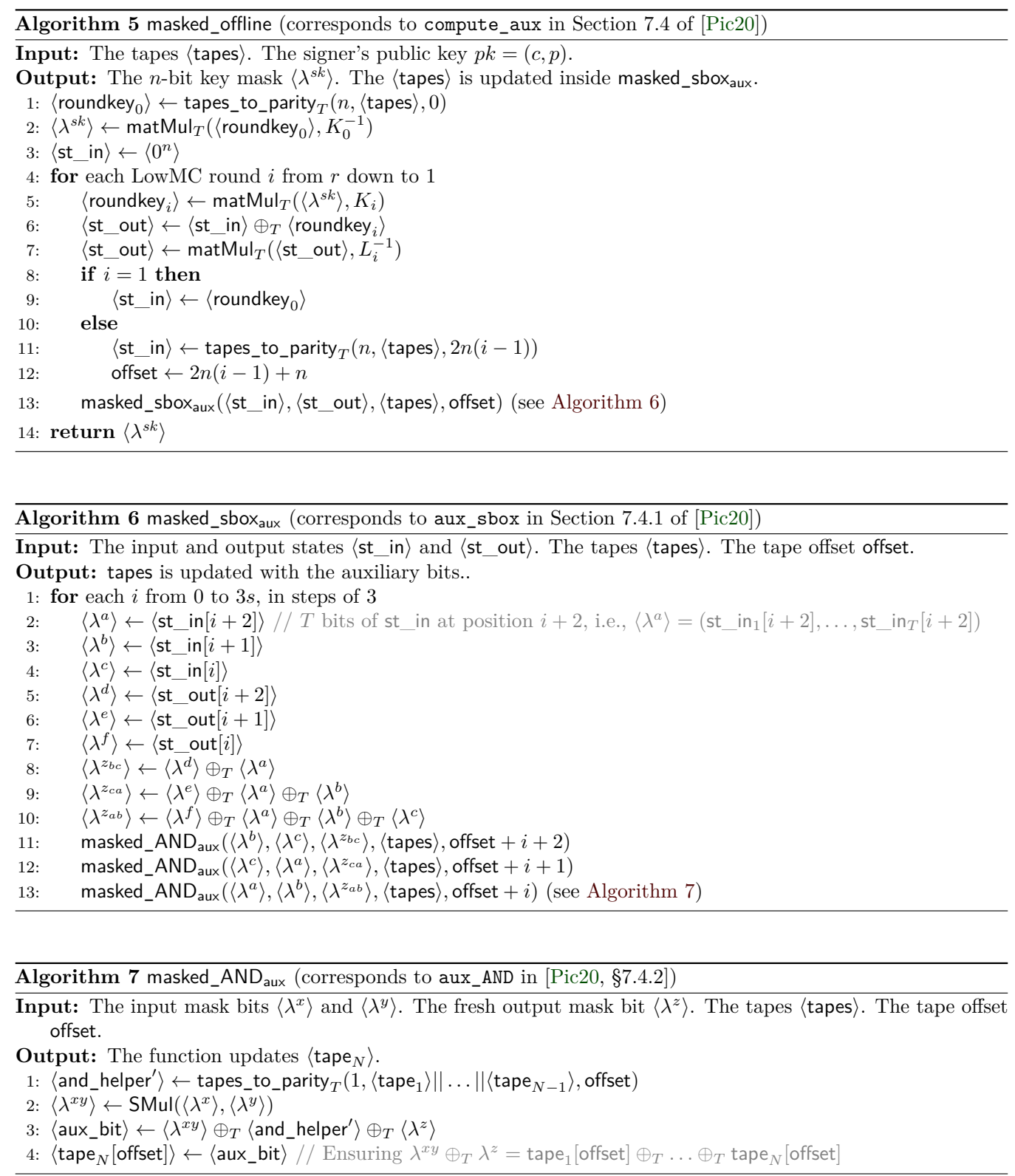

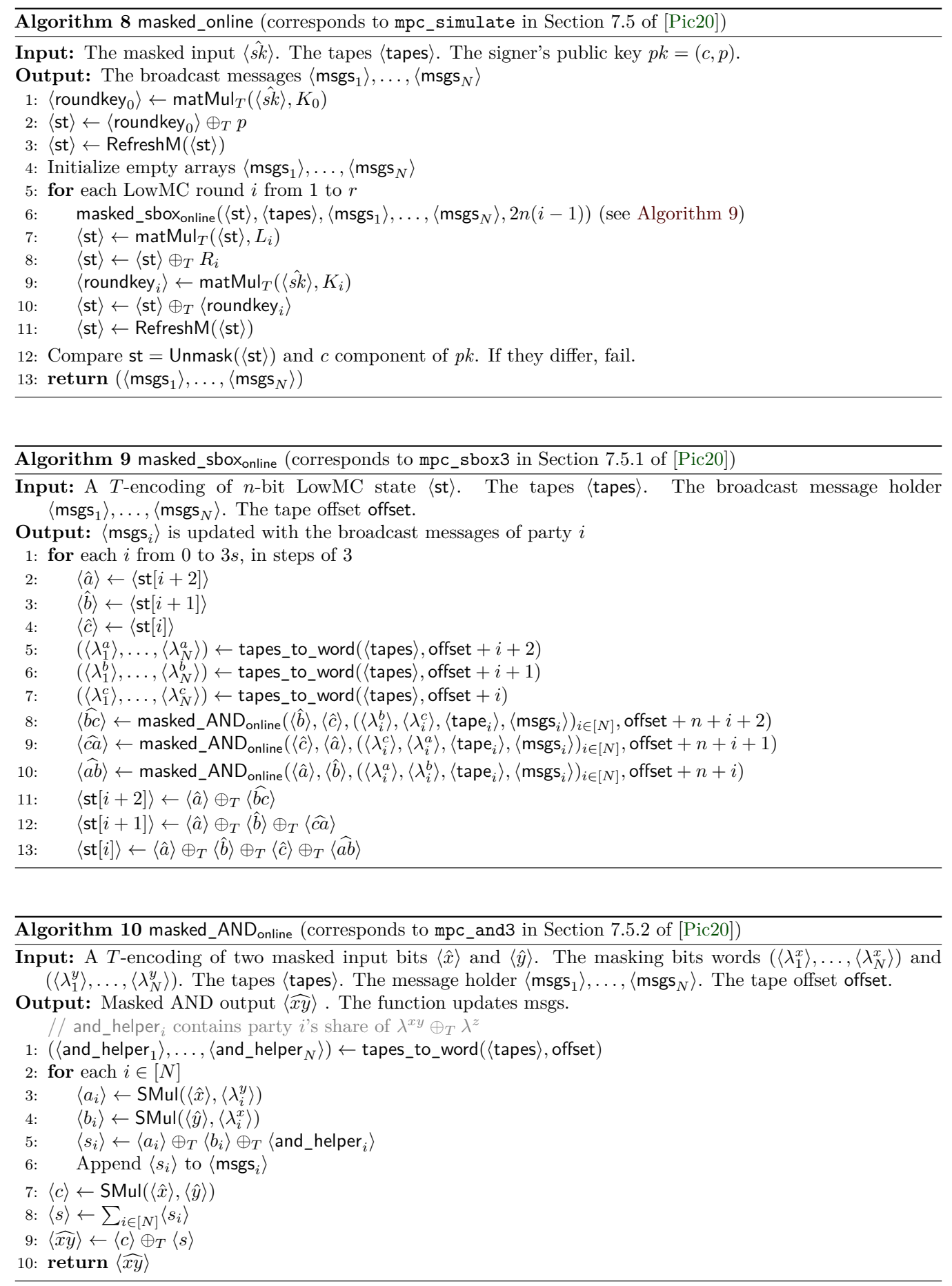


\section{Additional Gadgets}
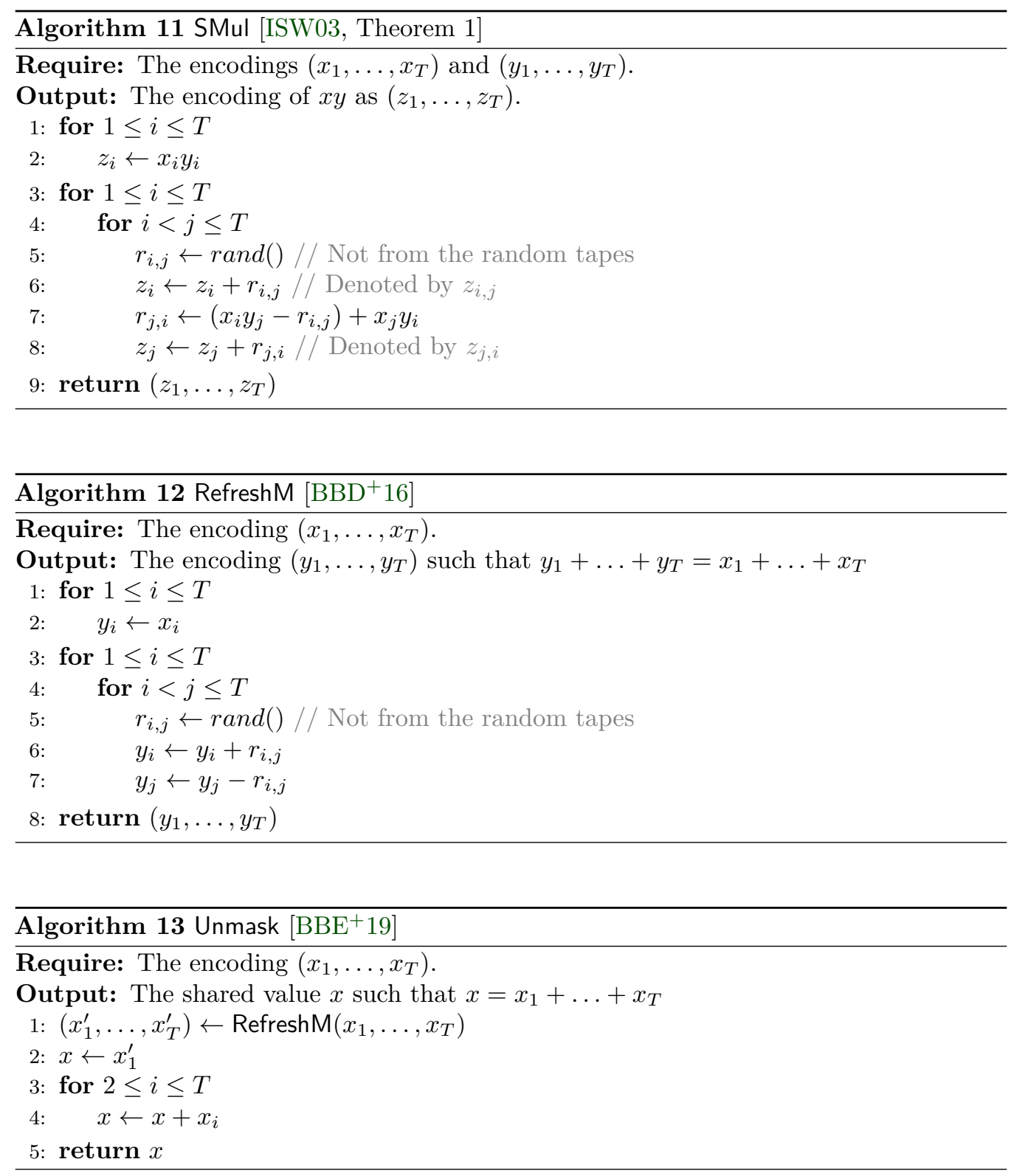

\section{E Specification of Unprotected Picnic3}

For completeness, we include the full specifications of Picnic3 signing adapted from [Pic20]. The notation is as defined elsewhere in the paper, and in the appendix on LowMC (Appendix F). 


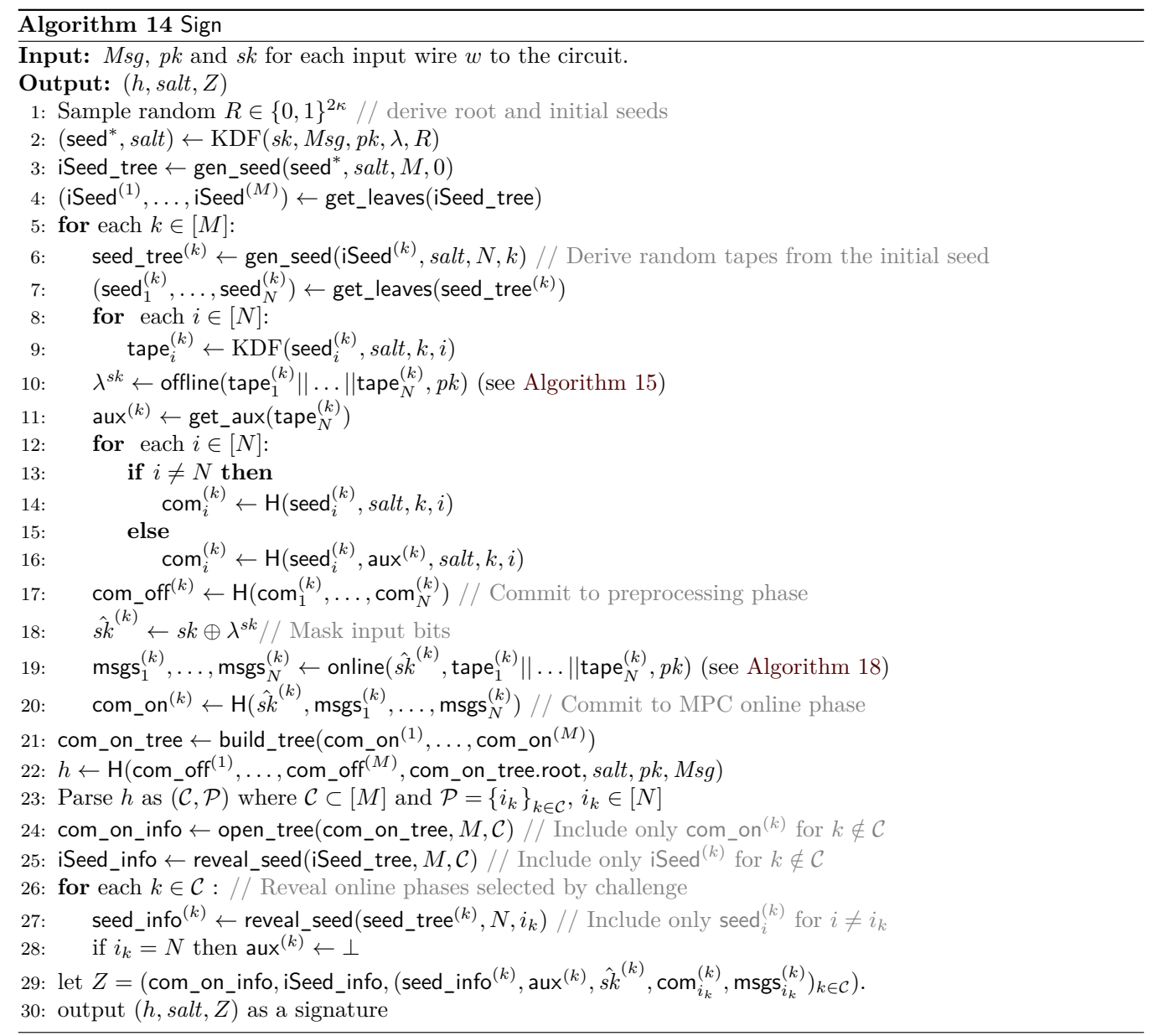



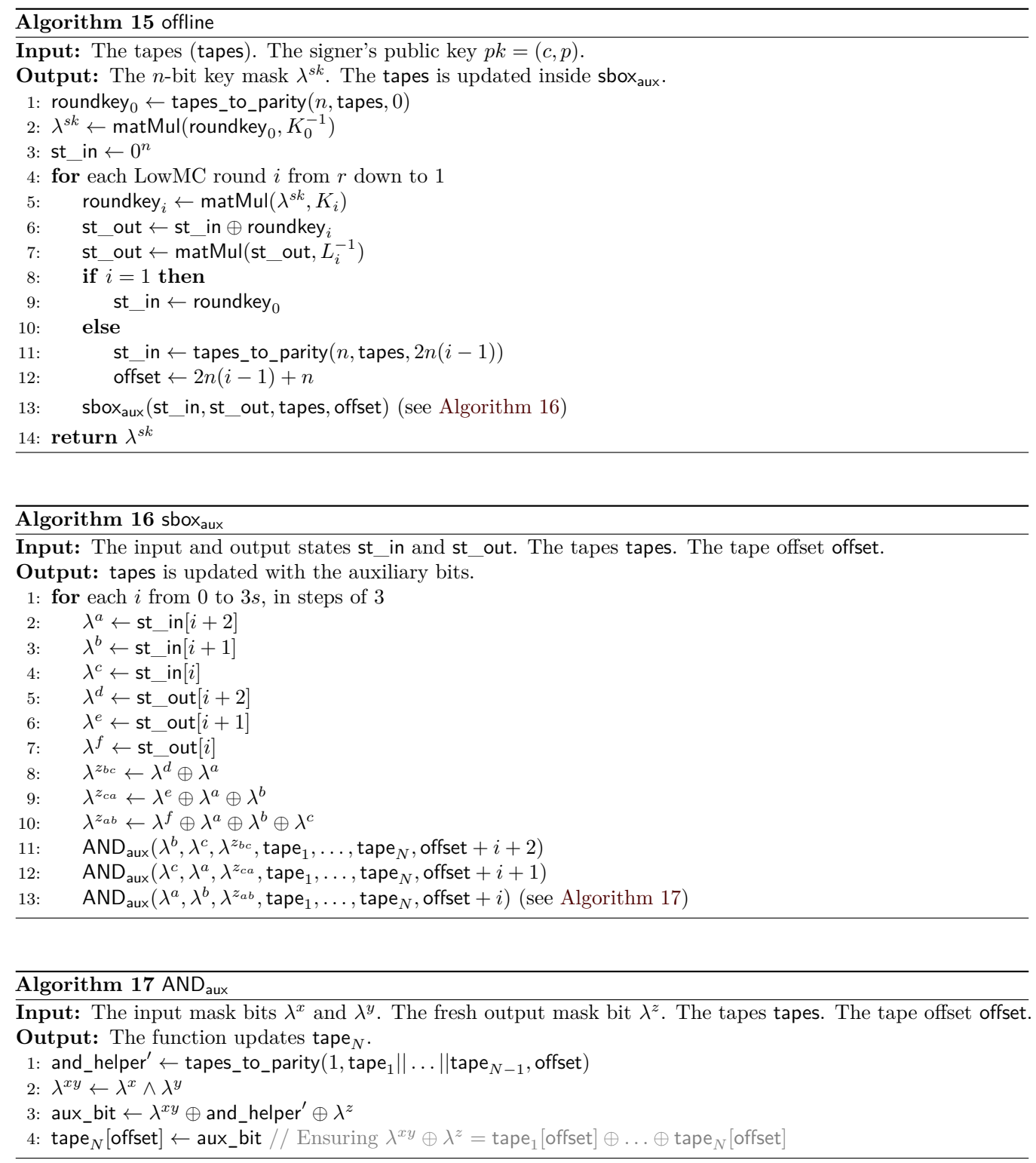

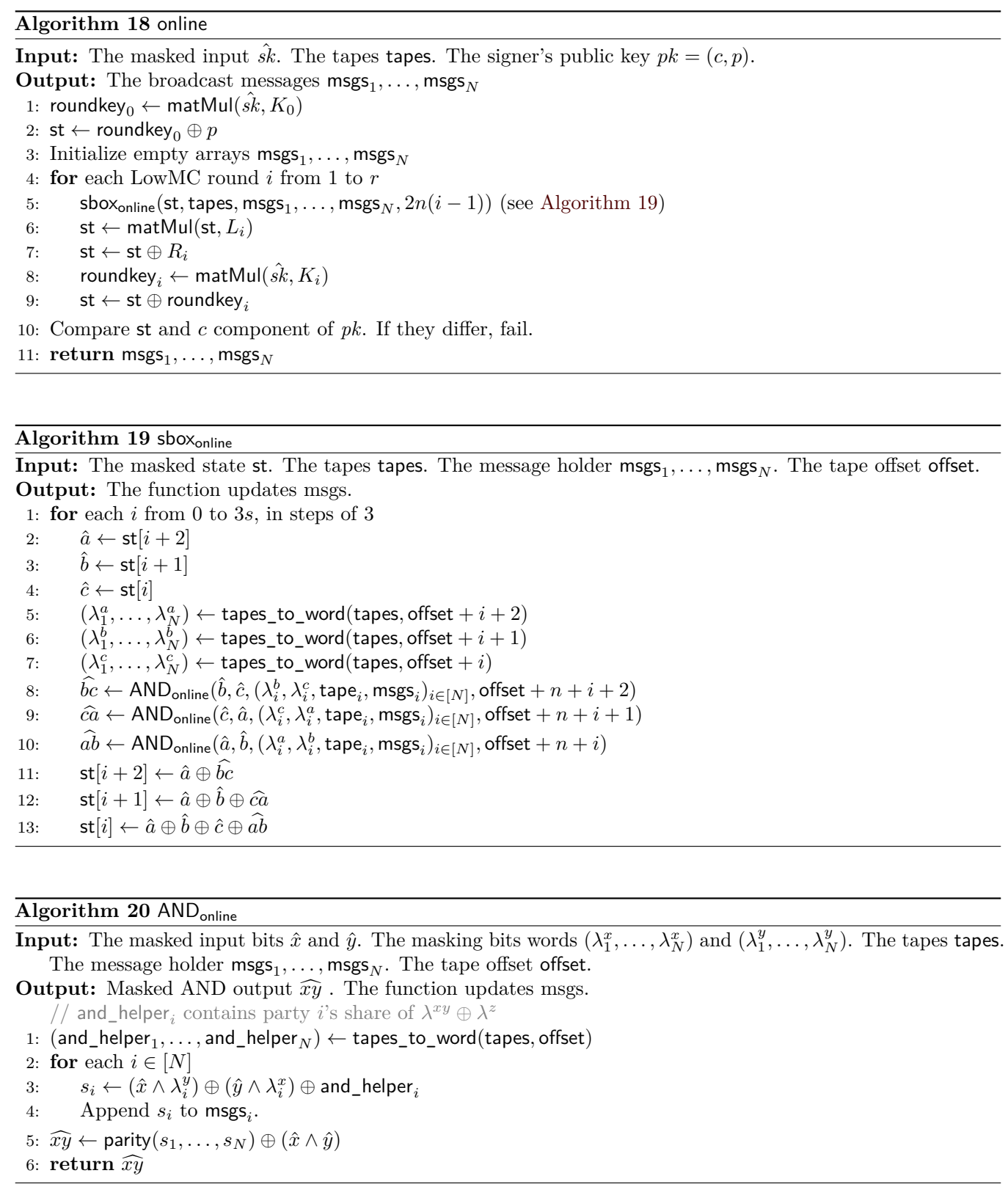

\section{F LowMC}

Low MC $\left[\mathrm{ARS}^{+} 15\right]$ is a parameterizable block cipher designed to have a small number of AND gates (low multiplicative complexity). In this work we assume the LowMC instances are those from the Picnic3 design; however, our analysis and countermeasures generalize easily to other choices of LowMC paramters.

Let $n$ be the block size and key size, $s$ be the number of S-boxes per round, and $r$ the number of rounds. For each LowMC instance, the spec defines random and independent

- round constants $R_{i} \in \mathbb{F}_{2}^{n}$,

- linear layer matrices $L_{i} \in \mathbb{F}_{2}^{n \times n}$ (of full rank), and 
- key matrices $K_{i} \in \mathbb{F}_{2}^{n \times n}$ for the computation of round keys.

There are $r$ round and linear layer constants $R_{i}, L_{i}$, and $r+1$ key matrices $K_{i}$. The matrices are invertible, and the Picnic3 implementation uses the inverses $K_{0}{ }^{-1}$ and $L_{i}{ }^{-1}$. LowMC keys are sampled uniformly at random from $\mathbb{F}_{2}^{n}$.

LowMC encryption starts by adding the first round key to the plaintext, which is followed by $r$ rounds. Each round key is generated by multiplying the key with the key matrix $K_{i}$. A single round of LowMC is composed of an S-box layer, a linear layer, addition with constants, and addition of the round key as shown in Algorithm 21. The S-box layer applies the same 3-bit S-box on the first $3 \cdot s$ bits of the state. The S-box is defined as $S(a, b, c)=(a \oplus b c, a \oplus b \oplus a c, a \oplus b \oplus c \oplus a b)$. The other layers only consist of $\mathbb{F}_{2}$-vector space arithmetic, all local operations in our MPC setting.

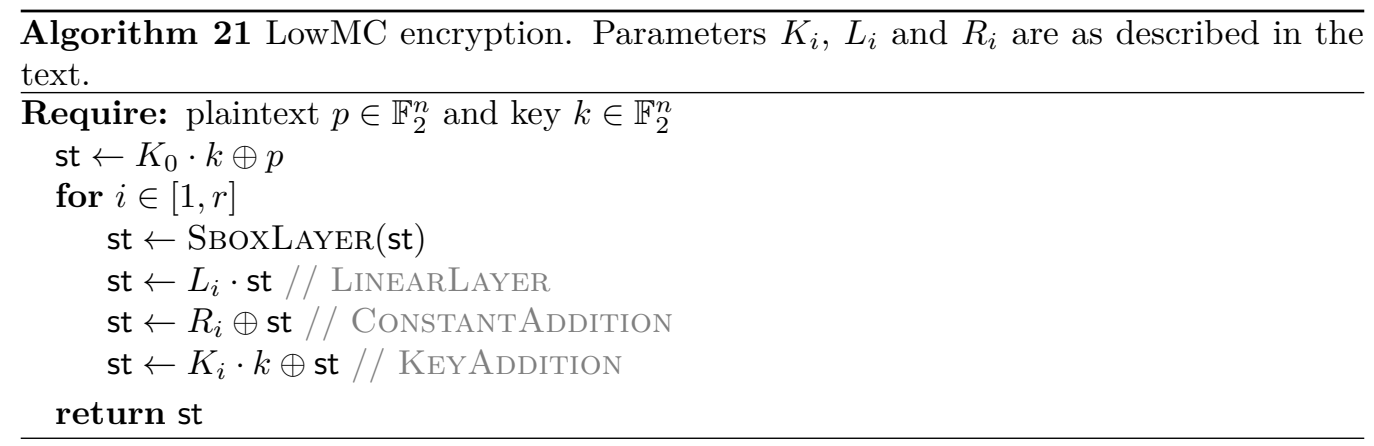

\section{G Additional Background on Security Notions for Masking Countermeasures}

In the following, we fix some finite field $(\mathbb{F}, 0,1,+,-, \cdot) .{ }^{5}$ As explained above, we are working in the $t$-probing model which allows an attacker to obtain the value of $t$ variables per run of the primitive. The most common technique to mitigate side-channel attacks is by encoding sensitive variables via an additive (or polynomial-based) secret sharing into $T>t$ parts. We say that a vector $\left(v_{j}\right)_{j \in[T]} \in \mathbb{F}^{T}$ is a $T$-encoding of $v:=\sum_{j \in[T]} v_{j}$. For readability, we often write $\langle v\rangle$ instead of $\left(v_{j}\right)_{j \in[T]}$. For a subset $I \subseteq[T]$, let $\langle x\rangle_{I}=\left(x_{i}\right)_{i \in I}$ and furthermore $\bar{I}=[T] \backslash I$. Variables are shared both to protect against side-channel attacks and as part of the MPC protocol. To distinguish between these situations, we call a sharing between parties in the MPC protocol a sharing and an encoding when the goal is to protect against side-channel attacks.

Without loss of generality, we only give the security definitions for circuits that receive a single encoded input $\langle x\rangle$ and produce a single encoded output $\langle y\rangle$. In the following, we use the terms circuit and gadget interchangeably. Consider a (possibly randomized) gadget $G$, which on input $x$ produces a value $y$ according to some probability distribution $G_{x}$. To ensure that the computation of $G$ does not leak any information, we modify it into a gadget $G^{\prime}$ that takes $\langle x\rangle$ as input and outputs $\langle y\rangle$ with $\operatorname{Pr}\left[G_{\langle x\rangle}^{\prime}=\langle y\rangle\right]=\operatorname{Pr}\left[G_{x}=y\right]$. Informally, we want to argue that the $t$ probes made by an attacker do not reveal any information about the sensitive input $x$. Assume that the attacker probes the values $\left\langle v^{(1)}\right\rangle_{I_{1}},\left\langle v^{(2)}\right\rangle_{I_{2}}$, $\ldots\left\langle v^{(k)}\right\rangle_{I_{k}}$ with $\sum_{j=1}^{k}\left|I_{j}\right| \leq t$. If $\langle x\rangle$ is a sufficiently random encoding (e.g. uniformly sampled) of $x$, then $\langle x\rangle_{I}$ does not reveal any information about $x$ for all $I \subsetneq[T]$. Now, if there exists a set $I \subsetneq[T]$ such that all $\left\langle v^{(j)}\right\rangle_{I_{j}}$ can be simulated from $\langle x\rangle_{I}$, this implies that the $\left\langle v_{I_{j}}^{(j)}\right\rangle$ do not contain any information about $x$.

\footnotetext{
${ }^{5}$ While ISW-style masking countermeasures are known to work in a ring setting, we focus on a field case since previous KKW-style protocols [KKW18, dDOS19, BN20, KZ20] are defined for circuits over a field.
} 
To formalize this intuition, we consider a distribution ensemble $\left\{D_{\langle x\rangle}\right\}_{\langle x\rangle \in \mathbb{F}^{T}}$. This ensemble is a probability distribution on $v \in \mathbb{F}^{t}$, capturing the probed variables of the attacker. We say that $\left\{D_{\langle x\rangle}\right\}_{\langle x\rangle}$ is perfectly simulatable from the indices $I \subseteq[T]$, if there is a probabilistic algorithm $S$ that, on input $\langle x\rangle_{I}$, has output distribution exactly $D_{\langle x\rangle}$, i.e., $\operatorname{Pr}\left[S\left(\langle x\rangle_{I}\right)=v\right]=\operatorname{Pr}\left[D_{\langle x\rangle}=v\right]$ for every $\langle x\rangle$.

Example 1. The simplest example for this notion of simulatability concerns projections. For example, if the ensemble $\left\{D_{\langle x\rangle}\right\}_{\langle x\rangle \in \mathbb{F}^{2}}$ is defined on the value $y=x_{1}$, it can easily be simulated from $I=\{1\}$, as the knowledge of $x_{1}$ is sufficient to simulate $y$.

Example 2. Consider the following distribution $\left\{D_{\langle x\rangle}\right\}_{\langle x\rangle \in \mathbb{F}^{2}}$ on the triple of values $\left(y_{1}, y_{2}, t\right)$ with $t=x_{1}+r, y_{1}=t \cdot x_{2}=\left(x_{1}+r\right) \cdot x_{2}$, and $y_{2}=x_{2}$. Here, $r$ is uniformly sampled from $\mathbb{F}$, independent from $\langle x\rangle$. This distribution ensemble is perfectly simulatable from $I=\{2\}$ : The value of $t$ can simply be simulated by sampling a random element from $\mathbb{F}$ without any knowledge on $x_{1}$. Now, both $y_{2}$ and $y_{1}$ can be simulated by using the knowledge about $x_{2}$ and the already simulated value $t$.

\section{G.1 Non-interference, Strong Non-interference, and Public Outputs}

The most basic security notion for a masking countermeasure is the $t$-privacy of a gadget $G$ [ISW03]. Informally, this means that the information provided by $t$ probes of outputs or intermediate variables can also be obtained by probing $t$ input variables, as long as the inputs are an encoding of $x$. While the idea behind the notion is relatively simple, it is unfortunately not composable as the output of a $t$-private gadget is not necessarily a truly uniform encoding. The composition of two $t$-private gadgets is thus not necessarily $t$-private. In order to remove the requirement that the inputs have a certain distribution, the notion of non-interference was introduced $\left[\mathrm{BBD}^{+} 15 \mathrm{a}\right]$.

Non-interference gets rid of the dependency of the uniformly encoded inputs, but a more subtle issue still prevents a composability result. To give an intuitive overview of this problem, consider a gadget $G$ with two sensitive inputs $\langle x\rangle$ and $\left\langle x^{\prime}\right\rangle$ and sensitive output $\langle y\rangle$. Non-interference now implies that for any $I_{y} \subsetneq[T]$, the values $\langle y\rangle_{I_{y}}$ can be simulated from $\langle x\rangle_{I_{x}}$ and from $\left\langle x^{\prime}\right\rangle_{I_{x^{\prime}}}$ for two sets $I_{x}, I_{x^{\prime}}$ of cardinality at most $\left|I_{y}\right|$. Now, if $G$ is used in another circuit, it might be the case that $x$ and $x^{\prime}$ are correlated (or even identical). Then $\langle x\rangle_{I_{x} \cup I_{x^{\prime}}}$ might reveal information about $x$. See e.g., $\left[\mathrm{BBD}^{+} 16\right]$ for a more detailed explanation. Hence, an even stronger notion, called strong non-interference was introduced $\left[\mathrm{BBD}^{+} 16\right]$, that guarantees a clear separation between input variables and output variables.

Definition $2\left(t\right.$-NI, $t$-SNI). Let $G$ be a gadget with inputs in $\mathbb{F}^{T}$ and $t<T$. Suppose that for any set of $t_{1}$ intermediate variables and any subset of $O$ of output indices with $t_{1}+|O| \leq t$, there exists a subset of indices $I$ such that the output distribution of the $t_{1}$ intermediate variables and the output variables $y_{\mid O}$ is perfectly simulatable from $I$. Then

(i) if $|I| \leq t_{1}+|O|$ we say $G$ is t-non-interfering ( $t$-NI), and

(ii) if $|I| \leq t_{1}$ we say $G$ is $t$-strong-non-interfering ( $t$-SNI).

Note that linear operations which can be performed share-wise (such as addition or multiplication by a constant) are trivially $t$-NI, as each computation on share $i$ can be simulated from the input share $x_{i}$.

Note that in SNI the size of the input set $I$ depends only on the intermediate variables. For a given set Int of $t_{1}$ intermediate variables and a subset $O$ of output indices with $t_{1}+|O| \leq t$, we say that the output variables $O^{\prime} \subseteq O$ that are simulatable without any knowledge about $I$ are (Int, $O, t$ )-input-ignorant. Hence, if no intermediate variable was probed, the output of the circuit is independent of its input (from observing at at most $t$ positions) and thus all subsets of at most $t$ outputs are input-ignorant. We will occasionally 
talk about the concrete distribution of these input-ignorant variables. If all (Int, $O, t$ )-inputignorant output variables of a $t$-SNI gadget $G$ are distributed according to a distribution $D$, we say that $G$ is t-strong-non-interfering (t-SNI) with output-distribution $D$.

Finally, the SNI notion guarantees that the composition of two $t$-SNI gadgets is $t$-SNI again. In Appendix D we recall two $t$-SNI-secure gadgets SMul and RefreshM that we use as building blocks of our masked KKW proof system. For the sake of completeness, we repeat the corresponding proposition from $\left[\mathrm{BBD}^{+} 16\right]$.

Lemma 3 (Proposition $4\left[\mathrm{BBD}^{+} 16\right]$ ). Let $C$ be a circuit built from gadgets $G_{1}, \ldots, G_{r}$ such that all $G_{i}$ are $t-N I$, all encodings are used at most once as input of a gadget call other than RefreshM. Then $C$ is $t$-NI. Moreover, $C$ is $t$-SNI if it is $t$-NI and all encodings corresponding to the outputs of $C$ are refreshed through RefreshM before output.

The commonly used term probing-security can either mean privacy $\left[\mathrm{BBC}^{+} 19\right]$ or noninterference [CGPZ16]. Classically, the non-interference notions only deal with gadgets where all of the inputs and outputs are sensitive. To also handle public, non-sensitive values, the notion of NI with public output ( $t$-NIo) was proposed in $\left[\mathrm{BBE}^{+} 18\right]$. As mentioned in $\left[\mathrm{BBE}^{+} 18\right.$, Lemma 1], if a gadget $G$ is $t$-NI secure it is also $t$-NIo secure for any public outputs. Clearly, the same claim also holds for $t$-SNI and $t$-SNIo. While the KKW-protocol also contains public variables, we are able to show the stronger guarantee of $t$-(S)NI.

\section{H Omitted Proofs}

In this section we give formal security proofs for the SNI security of Algorithm 22 and 23.

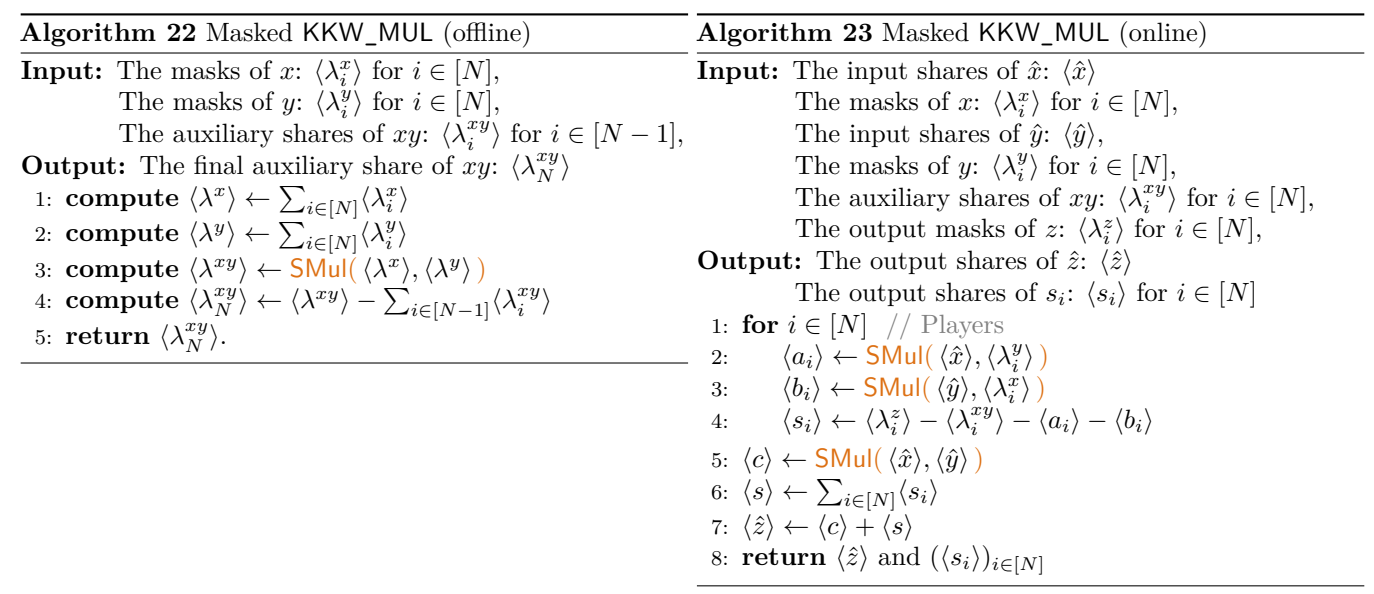

\section{H.1 Proof of Lemma 1}

Proof. We thus need to show that for any set of $t<T$ intermediate variables and any subset $\mathcal{O} \subset\left[\hat{z}_{1}, \ldots, \hat{z}_{T}\right]$ of output shares such that $t+|\mathcal{O}|<T$, for each input variable $v$, there is an input set $I_{v}$ with $\left|I_{v}\right| \leq t$ such that the $t$ intermediate variables and the output variables $\left\langle\lambda_{N}^{x y}\right\rangle_{\mathcal{O}}$ can be perfectly simulated from these input sets.

Both the computation of $\left\langle\lambda^{x}\right\rangle$ and $\left\langle\lambda^{y}\right\rangle$ are straightforward and can be simply simulated, as they are linear operations. Whenever one of the terms involved in the computation of $\left\langle\lambda^{x y}\right\rangle \leftarrow \operatorname{SMul}\left(\left\langle\lambda^{x}\right\rangle,\left\langle\lambda^{y}\right\rangle\right)$ is probed, we add the corresponding values from the proof of the SNI-security (found e.g. in $\left[\mathrm{BBD}^{+} 16\right.$, Proposition 2]) of SMul( ) to the input sets $I_{v}$. The result $\left\langle\lambda^{x y}\right\rangle$ can be simulated without any input as $\operatorname{SMul}()$ is SNI. To simulate the output later on, we add all $\lambda_{i, j}^{x y}$ to the inputs $I_{v}$.

Finally, the computation of $\left\langle\lambda_{N}^{x y}\right\rangle$ is again linear. 
For the output, suppose that $\lambda_{N, j}^{x y}$ was probed. There are two cases to consider: If $\lambda_{j}^{x y}$ was probed, the inputs $I_{v}$ already contains all $\lambda_{i, j}^{x y}$ and we can thus simulate $\lambda_{N, j}^{x y}$ perfectly. If $\lambda_{j}^{x y}$ was not probed, $\lambda_{j}^{x y}$ looks like a uniformly random element from $\mathbb{F}$ that is not used anywhere else, as it is produced by a $t$-SNI gadget with uniform output-distribution. We can thus uniformly sample a random element $r \in \mathbb{F}$ and replace $\lambda_{N, j}^{x y}$ by $r$. This implies strong non-interference.

\section{H.2 Proof of Lemma 2}

Proof. We thus need to show that for any set of $t<T$ intermediate variables and any subset $\mathcal{O}$ of output shares such that $t+|\mathcal{O}|<T$, for each input variable $v$, there is an input set $I_{v}$ with $\left|I_{v}\right| \leq t$ such that the $t$ intermediate variables and the output variables indexed by $\mathcal{O}$ can be perfectly simulated from these input sets. To show this, we go through all variables of the algorithm and explain for all input variables $v$ which indices are added to $I_{v}$.

Whenever one of the terms involved in the SMul( )-computation for a term $a_{i, j}, b_{i, j}$, or $c_{j}$ is probed, we add the corresponding values from the proof of the strong non-interference of SMul( ) to the input sets $I_{v}$. Note that no inputs need to be added to $I_{v}$ if $a_{i, j}, b_{i, j}$, or $c_{j}$ were probed, as they are the result of a $t$-SNI gadget.

Whenever $s_{i, j}$ or a sub-term of $s_{i, j}$ is probed, we add the variables corresponding to $a_{i, j}, b_{i, j}, \lambda_{i, j}^{z}$, and $\lambda_{i, j}^{x y}$ to the input sets $I_{v}$. This clearly allows us to simulate all $s_{i^{\star}, j}$ and all sub-terms perfectly.

Whenever a sum $\sum_{i=1}^{i^{\prime}} s_{i, j}$ (including $s_{j}$ itself) is probed, we distinguish two cases. If $s_{1, j}, s_{2, j}, \ldots, s_{i^{\prime}, j}$ were all probed, we can simply simulate the complete sum. Otherwise, there is a term $s_{i^{\star}, j}$ with $i^{\star} \in\left\{1, \ldots, i^{\prime}\right\}$ such that $s_{i^{\star}, j}$ was not probed. As $s_{i^{\star}, j}$ is the only place where $a_{i, j}$ is used, we make use of the fact that $a_{i, j}$ is constructed by an $t$-SNI gadget with uniform output-distribution. In other words, this means that $a_{i, j}$ looks like a uniformly random element from $\mathbb{F}$ that is not used anywhere else. We can thus uniformly sample a random element $r \in \mathbb{F}$ and replace the complete sum $\sum_{i=1}^{i^{\prime}} s_{i, j}$ by $r$. Note that in the previous argument, we did not add anything to $I_{v}$.

Finally, whenever $\hat{z}_{j}$ is probed, we simply simulate $s_{j}$ and $c_{j}$. As $c_{j}$ is the result of a $t$-SNI gadget, we can simulate it without needing to add anything to the input sets $I_{v}$. As shown in the discussion about $s_{j}$, we can also simulate it without needing to add anything to the input sets $I_{v}$. This implies strong non-interference. 